Published in final edited form as:

Nat Protoc. 2018 September ; 13(9): 2102-2119. doi:10.1038/s41596-018-0036-3.

\title{
PEG-4MAL hydrogels for human organoid generation, culture, and in vivo delivery
}

\author{
Ricardo Cruz-Acuña ${ }^{1,2,8}$, Miguel Quirós ${ }^{3,8}$, Sha Huang ${ }^{4,5,6}$, Dorothée Siuda ${ }^{3}$, Jason R. \\ Spence $4,5,6,8,{ }^{*}$, Asma Nusrat ${ }^{3,8,{ }^{*}}$, Andrés J. García $2,7,8,{ }^{*}$ \\ ${ }^{1}$ Wallace H. Coulter Department of Biomedical Engineering, Georgia Institute of Technology, \\ Atlanta, GA, USA \\ ${ }^{2}$ Parker H. Petit Institute for Bioengineering and Biosciences, Georgia Institute of Technology, \\ Atlanta, GA, USA \\ ${ }^{3}$ Department of Pathology, University of Michigan, Ann Arbor, MI, USA \\ ${ }^{4}$ Division of Gastroenterology, Department of Internal Medicine, University of Michigan Medical \\ School, Ann Arbor, MI, USA \\ ${ }^{5}$ Department of Cell and Developmental Biology, University of Michigan Medical School, Ann \\ Arbor, MI, USA \\ ${ }^{6}$ Center for Organogenesis, University of Michigan Medical School, Ann Arbor, MI, USA \\ ${ }^{7}$ George W. Woodruff School of Mechanical Engineering, Georgia Institute of Technology, Atlanta, \\ GA, USA
}

\section{Abstract}

In vitro differentiation of human pluripotent stem cell (hPSC)-derived organoids (HOs) facilitates the production of multicellular three-dimensional structures analogous to native human tissues. Most current methods for the generation of HOs rely on Matrigel, a poorly defined basement membrane derivative secreted by Engelbreth-Holm-Swarm mouse sarcoma cells, limiting the potential use of HOs for regenerative medicine applications. Here, we describe a protocol for the synthesis of a fully defined, synthetic hydrogel that supports the generation and culture of HOs. Modular, cell-encapsulating hydrogels are formed from a four-armed poly(ethylene glycol) macromer that has maleimide groups at each terminus (PEG-4MAL) and is conjugated to cysteine-containing adhesive peptides and cross-linked via protease-degradable peptides. The protocol also includes guidelines for the localized in vivo delivery of PEG-4MAL hydrogel-

\footnotetext{
Reprints and permissions information is available at www.nature.com/reprints.

"Corresponding Author: spencejr@umich.edu; anusrat@med.umich.edu; andres.garcia@me.gatech.edu.

${ }^{8}$ These authors contributed equally to this work: Ricardo Cruz-Acuña, Miguel Quirós.

Author contributions

R.C.-A. and M.Q. conducted all experiments, collected data, and performed data analyses. S.H. performed the culture and differentiation of hPSCs into intestinal spheroids. D.S. assisted with in vivo HIO delivery to mouse colonic wounds. A.N., A.J.G., and J.R.S. conceptualized and designed the project and experiments. R.C.-A., A.J.G., A.N., J.R.S., and M.Q. wrote the manuscript. Competing interests

R.C.-A., M.Q., J.R.S., A.N., and A.J.G. are inventors on a patent application related to this work and co-owned by the Georgia Tech Research Corp. and the University of Michigan. The remaining authors declare no competing interests.

Supplementary information is available for this paper at https://doi.org/10.1038/s41596-018-0036-3.
} 
encapsulated HOs to injured mouse colon. The PEG-4MAL hydrogel supports the engraftment of the HOs and accelerates colonic wound repair. This culture and delivery strategy can thus be used to develop HO-based therapies to treat injury and disease. Hydrogel and tissue preparation and subsequent encapsulation can be performed within 2.5-3.5 h. Once HOs have been cultured in synthetic hydrogels for at least $14 \mathrm{~d}$, they can be prepared and delivered to the mouse colon in under $5 \mathrm{~h}$.

\section{Introduction}

Human pluripotent stem cell (hPSC)-derived organoids (HOs) are formed after the directed differentiation of hPSCs into three-dimensional (3D) structures (spheroids), followed by culture in specific in vitro culture conditions that induce further differentiation of cells ${ }^{1,2}$. These multicellular 3D structures recapitulate important features of epithelial and mesenchymal tissues, making them valuable tools in the study of a range of cellular processes $^{3,4}$. In this context, protocols have been developed for the in vitro generation of HOs modeling different tissues, for example, intestine ${ }^{1,2}$, lung $^{5,6}$, brain $^{7}$, retina ${ }^{8}$, and kidney ${ }^{9}$. Such HOs provide powerful platforms for modeling human organ development and chronic diseases such as cancer and inflammatory bowel disease ${ }^{10}$. Furthermore, HOs can potentially serve as tissue sources for patient-specific regenerative therapies ${ }^{4}$.

Most currently available protocols for organoid generation require encapsulation of spheroids within biologically derived materials such as Matrigel, which are not well characterized and thus exhibit considerable lot-to-lot variability, poor experimental control, and the inability to decouple their biochemical and biophysical properties ${ }^{11-13}$. In addition, in the case of Matrigel, the fact that this material is derived from cancerous murine cells limits its translational potential ${ }^{14,15}$. Fully defined, synthetic hydrogels that present tunable physicochemical properties are promising alternatives to current biologically derived matrices for organoid culture, as they can mediate innate cellular responses via presentation of bioactive motifs that promote cell-matrix-adhesive interactions and cell-directed matrix degradation ${ }^{15,16}$.

Here, we describe a protocol for the synthesis of a fully synthetic PEG-4MAL hydrogel that supports the robust and highly reproducible in vitro generation of HOs from human induced pluripotent stem cell (hiPSC)- and embryonic stem cell (hESC)-derived spheroids without the need for Matrigel encapsulation. We have previously used this hydrogel to generate and culture human intestinal organoids (HIOs) and human lung organoids (HLOs) ${ }^{16}$.

\section{Development and advantages of the protocol}

Hydrogels are water-swollen, cross-linked polymer networks with attractive mechanical and biochemical properties for a variety of biomedical and biotechnological applications ${ }^{14}$. The fully defined synthetic hydrogel system described in this protocol is based on a four-armed, maleimide-terminated PEG-4MAL macromer that is engineered to present elements and traits inspired by extracellular matrices, such as cell-adhesion peptides and matrix sensitivity to cell-secreted proteases. Although many synthetic hydrogel systems have been developed to mimic the properties of natural extracellular matrices, the PEG-4MAL hydrogel platform 
exhibits substantial advantages over other synthetic hydrogels (e.g., poly(acrylic acid)- and poly(vinyl alcohol)-based hydrogels) developed to date, including their intrinsic low-protein adsorption, the stoichiometric incorporation of cell-adhesive peptides (and other biological signals), minimal inflammatory profile, and history of safe in vivo use ${ }^{17,18}$. In addition, PEG-4MAL hydrogel polymerization chemistry presents increased cytocompatibility over free-radical-initiated polymerization, and improved cross-linking efficiency over acrylate (PEG-4A) and vinylsulfone (PEG-4VS) polymerization ${ }^{16-19}$. Finally, although a complex PEG-based hydrogel has been previously developed for in vitro generation of murine intestinal organoids ${ }^{15}$, this protocol is advantageous because it describes a single PEG-4MAL hydrogel formulation that does not require animal-derived components (e.g., laminin-111) for the in vitro generation and in vivo delivery of human organoids, establishing its potential for regenerative medicine applications ${ }^{16}$.

The tunability of PEG-4MAL hydrogel properties enables the study of the independent contributions of the biophysical and biochemical properties of the matrix in both single-cell and multicellular programs. For instance, using this platform, we showed that normal cyst growth, polarization, and lumen formation of renal epithelial Madin-Darby canine kidney (MDCK) cells could take place only within a narrow range of values for the elasticity of the matrix, required a threshold level of cell-directed matrix degradability, and were subject to a tight regulation imposed by the adhesive peptide density ${ }^{19}$. Furthermore, we have recently reported that both the in vitro generation and culture of HIOs from hPSC-derived spheroids and the culture of HLOs were dependent on the mechanical and biochemical properties of the PEG-4MAL hydrogel ${ }^{16}$. The PEG-4MAL hydrogel system can thus provide a robust platform for the in vitro generation and culture of different types of HOs, hence, facilitating the study of the contributions of the extracellular matrix to human organ development, differentiation, and function. We used an engineered hydrogel (storage modulus $(G): 100$ Pa; 4.0\% (wt/vol), 20-kDa PEG-4MAL; $2.0 \mathrm{mM}$ RGD (GRGDSPC); and GPQ-W (GCRDGPQGIWGQDRCG) cross-linker) to support the in vitro generation of HIOs from hPSC-derived spheroids and culture of $\mathrm{HLOs}^{5}$, establishing its potential to generate and maintain different types of HOs ${ }^{16}$. The modular design of the PEG-4MAL hydrogel system facilitates independent optimization of the physicochemical properties of the synthetic matrix, and hence enables the identification of an optimal engineered formulation for $\mathrm{HO}$ generation. The mechanical properties of the synthetic matrix can be controlled by varying the polymer density, independently of the matrix's biochemical properties (adhesive peptide type or density) ${ }^{16,18,19}$. Conversely, the biochemical characteristics of the material can be modified by changing the adhesive peptide type (e.g., laminin- or collagen-derived peptides) or density, independently of the material's mechanical properties. Modifications of the matrix properties were proven to have a direct effect on the epithelial morphogenesis of different cell systems and on spheroid development into HIOs ${ }^{16,19}$. Therefore, in addition to the inherent potential of the engineered hydrogel, its modular design supports the adaptation of this synthetic material to facilitate the generation and culture of different types of HOs.

An additional advantage of the hydrogel is the cost: the total cost of hydrogel components necessary to produce a total of $10 \mathrm{~mL}$ of fully cross-linked synthetic hydrogel is $\sim \$ 140$, whereas $10 \mathrm{~mL}$ of Matrigel costs $\sim \$ 305$. Therefore, PEG-4MAL hydrogels serve as an in vitro synthetic platform that can be modulated to support different cellular developmental 
programs, such as $\mathrm{HO}$ development, while overcoming the limitations of biologically derived materials such as Matrigel.

In addition, use of the PEG-4MAL hydrogel affords tunable reaction time scales for in situ gelation for in vivo applications. This synthetic hydrogel system has been successfully used as a delivery vehicle, demonstrating rapid gelation and integration at the transplantation site. This hydrogel system has also been shown to support the viability of encapsulated cells and to readily undergo the cell-mediated degradation that promotes engraftment of delivered cells into the host tissue ${ }^{20,21}$. We have demonstrated that injection of HIOs and the liquid precursors of the hydrogel into mucosal wounds in the murine colon resulted in in situ formation of a polymerized hydrogel that supported localized organoid engraftment and accelerated wound repair ${ }^{16}$. We have demonstrated that the presence of the delivery hydrogel was required for HIO engraftment at the implantation site ${ }^{16}$. Furthermore, the base macromer exhibits minimal toxicity and inflammation in vivo and is rapidly excreted via the urine. These traits are advantageous when considering the safety of hydrogels and their possible translation to the clinic ${ }^{20}$. Therefore, this delivery strategy could be used as a starting point in the development of HO-based tissue-replacement therapies in which HOs are engrafted directly into injured or diseased organs, for example, as an approach to treat human gastrointestinal diseases associated with intestinal epithelial wounds (e.g., inflammatory bowel disease).

\section{Limitations of the protocol}

An important aspect of this engineered hydrogel platform is its rapid reaction kinetics, which may result, if mixing is not conducted properly, in the formation of an inhomogeneous gel that presents variability in its physicochemical properties. Therefore, to avoid inhomogeneities in the hydrogel properties due to premature cross-linking, the functionalized macromer and cross-linker solutions may need to be delivered separately and mixed at the in vivo delivery site, or to be mixed before rapid delivery into the in vivo site. Furthermore, for adhesive ligands and cross-linking peptides to be incorporated into PEG-4MAL hydrogels, these molecules must have free thiol groups that can react with the maleimide moieties of the hydrogel backbone. Therefore, these signaling sequences might need to be custom-synthesized to contain a free thiol group (e.g., a cysteine residue) that enables the chemical coupling into PEG-4MAL hydrogels to take place.

\section{Experimental design}

In this section, we highlight some of the things that should be considered before embarking on an experiment using PEG-4MAL hydrogels, such as sample size, modifications required for use on specific HOs, and choice of animal model. In the Procedure, we describe how to prepare PEG-4MAL hydrogels and use them to generate and passage human organoids. We also provide specific instructions for the encapsulation of human organoids and their administration into mouse colon with a mucosal wound. We hope that the latter procedure will be helpful to users wishing to adapt the protocol to administer hydrogels to test alternative potential therapeutic systems or basic science research questions. 


\section{Considerations to ensure reproducible experiments}

PEG-4MAL hydrogels have previously been demonstrated to be a reproducible platform for the in vitro generation and in vivo delivery of $\mathrm{HOs}^{16}$. The reproducibility of the synthetic hydrogel was established with a sample size of at least four hydrogels per condition (20-30 spheroids/hydrogel or 2-4 HOs/hydrogel) for in vitro experimentation, and four mice per condition (four to five injections per mouse) for in vivo experimentation, with the premise that an outcome present in all hydrogels or animals under a specific condition will reveal the population behavior submitted to this given condition ${ }^{16}$. In addition, for a particular hydrogel formulation, consistent biophysical and biochemical properties, as well as the resulting spheroid/HO responses, were observed across independent experimental runs performed on different days, demonstrating high reproducibility. For all in vitro experiments, if making more than one functionalized PEG-4MAL precursor solution, it is recommended to allocate all human spheroids or HO suspension to one tube (as indicated in the protocol) and to ensure proper mixing before mixing with the hydrogel precursor solutions, to ensure a random distribution of the biological tissue. No specific randomization scheme is recommended for in vivo delivery of HOs, although randomization of samples is highly recommended. For in vivo experiments, control groups should be included based on the nature of the study; for instance, to study HIO engraftment in the colon of mice via injection of HIOs with hydrogel precursor solutions, we have included the following control groups: injection of only HOs and injection of only the hydrogel, as previously described ${ }^{16}$. Finally, to avoid research bias, it is recommended that the researcher(s) performing the experiments is(are) different from the researcher (s) processing and analyzing the experimental results.

\section{Generation, culture, and passage of hPSC-derived spheroids and HOs}

Efficient methods have been developed to generate HOs from in vitro hPSC cultures through direct differentiation protocols that are specific to the developmental program of the tissue origin of interest ${ }^{4}$. During the initial differentiation stages of hPSCs, human spheroids arise (by budding from the hPSC monolayer) and detach. To generate HOs, the detached, floating spheroids are collected and transferred to 3D culture, most commonly in Matrigel, where they further differentiate and expand into HOs, giving rise to several differentiated tissuespecific cellular populations. The generated HOs can be further cultured and passaged in the 3D culture environment for research purposes that encompass the study of organ development and tissue-specific disease progression ${ }^{4}$. However, fully defined, PEG-4MAL hydrogels that present tunable physicochemical properties are a promising alternative that overcomes several limitations of previous organoid culture matrices for translational medicine applications ${ }^{16}$.

This protocol provides guidelines for engineering a PEG-4MAL hydrogel for the in vitro generation of the desired HO. PEG-4MAL is used in place of Matrigel to encapsulate floating 3D spheroids generated from hPSC cultures by directed differentiation (e.g., midgut and hindgut spheroids for the generation of HIOs as previously described ${ }^{1,2,16}$ ). In addition, the synthetic matrix can be used for the continuous 3D culture and passaging of hPSCderived HOs that were generated using the synthetic material or using Matrigel (e.g., HIOs generated in Matrigel for at least $14 \mathrm{~d}$, as previously described ${ }^{1,2,16}$ ). 
Because this protocol is intended for the generation and culture of hPSC-derived HOs, the passaging steps provided here differ from trypsin-based passage protocols used for primary tissue-derived organoids ${ }^{13,22}$. For instance, owing to the mesenchymal layer and the large size of HIOs, these organoids cannot be passaged by trypsin using the methods developed for organoids derived from primary tissue. Instead, HIO must be passaged by manual cutting $^{2}$, as described in this protocol. Given that manual cutting had previously been used for HIO cultures grown in Matrigel $^{2}$, the procedure and time required to manually cut organoids into halves are independent of the matrix used and do not represent a limitation of the synthetic hydrogel. For hPSC-derived human organoids that do not require manual cutting, such as HLOs ${ }^{5}$, this specific step can be skipped.

\section{Adapting the hydrogels for different $\mathrm{HO}$ systems}

Hydrogels are generated by conjugating the PEG-4MAL macromer to an adhesive peptide to form a functionalized PEG-4MAL macromer (Fig. 1). The functionalized PEG-4MAL macromers are mixed with hPSC-derived spheroids or HOs and then cross-linked with a protease-degradable peptide for $20 \mathrm{~min}$ before adding growth medium to the polymerized hydrogel (Fig. 2) ${ }^{16}$.

An attractive feature of the PEG-4MAL hydrogel system is the ability to independently tune its biophysical and biochemical properties, which allows for its adaptation to the culture of different types of HOs. The macromer size (e.g., 10 versus $20 \mathrm{kDa}$ ) and polymer density can be modified to tune the density of cross-links within the hydrogel, which translates to changes in its biophysical properties; moreover, the type and density of adhesive and crosslinking peptides can be modulated to confer specific biochemical properties on this synthetic matrix. For instance, variations of PEG-4MAL polymer density directly control its stiffness ${ }^{16,18,19}$ ( $G$, a biophysical property), independently of its adhesive peptide type and density (2.0 mM RGD, a biochemical property) (Fig. 3). Given a constant adhesive peptide concentration, when the PEG-4MAL polymer density is increased (decreased), the number of additional (or fewer) maleimide moieties must be compensated for by a higher (or lower) concentration of cross-links (e.g., GPQ-W cross-linking peptide), which in turn increases (or decreases) the matrix stiffness. Therefore, in this hydrogel system, the mechanical properties (e.g., elastic modulus, or the related $G$ value determined by rheometry) are determined by the density of cross-links in the hydrogel network. Matrix stiffness is an important biophysical property to consider when designing a synthetic cellular matrix for the type of $\mathrm{HO}$ of interest, as we have previously shown that different biophysical cues (4 and 6\% (wt/ vol) PEG-4MAL polymer density, Fig. 3) support the morphogenesis programs of different epithelial cell lines (MDCK and Caco-2, respectively) ${ }^{19}$. Therefore, PEG-4MAL polymer density can be modified to tune the density of cross-links and identify a matrix stiffness that provides the physical support and promotes the essential mechanosignals for the generation and culture of the $\mathrm{HO}$ of interest. For reference, we have previously shown that PEG-4MAL polymer density controls HIO generation from hPSC-derived intestinal spheroids and influences the prolonged viability of HIOs generated in Matrigel ${ }^{16}$.

The functionalization of the PEG-4MAL macromer using adhesive peptides is an important biochemical property to consider when designing a synthetic cellular matrix that best 
resembles the biochemical environment of the native tissue of interest ${ }^{14}$. In this context, given a constant density of PEG-4MAL, the hydrogel macromer can be functionalized with different types and densities of cysteine-terminated adhesive peptides (e.g., RGD or lamininor collagen-derived peptides) to modify the biochemical characteristics of the material, considering that all unreacted maleimide groups remaining are cross-linked. For reference, we have previously shown that the adhesive peptide type has a substantial effect on HIO viability ${ }^{16}$, and that adhesive peptide (RGD) density markedly regulates cyst polarity and lumen phenotypes of epithelial cells ${ }^{19}$. Finally, the capacity of cells to modify their microenvironment via matrix degradation is essential for tissue remodeling and homeostasis ${ }^{14,23}$. In this context, given a constant density of PEG-4MAL, and a fixed type and density of adhesive peptide, the level of hydrogel degradability by proteases can be modulated by varying the ratio between a relatively fast-degrading (e.g., GPQ-W) and a slow- or nondegrading (e.g., GPQ-A ${ }^{19}$ or DTT ${ }^{16}$, respectively) cross-linking agent. Therefore, when designing a PEG-4MAL hydrogel matrix for the HO of interest, it is important to consider the use of a cross-linking peptide that is sensitive to the proteases expressed by the HO. We have previously demonstrated that prolonged survival of HIOs requires a degradable matrix cross-linked with the collagen-derived GPQ-W peptide ${ }^{16}$, and that normal epithelial cyst polarity and lumen formation required a threshold level (80\% fast-degrading GPQ-W) of protease-directed matrix degradability ${ }^{19}$. Finally, as in a previous report, users can evaluate the suitability of the synthetic hydrogel for the generation of HIOs by assessing human intestinal spheroid/HIO viability at different time points, proliferation, growth, and morphological changes of the HIO structure ${ }^{16}$.

This protocol describes a synthetic hydrogel platform that can be established to support the in vitro generation and culture of different types of HOs via modulation of its biophysical and biochemical matrix properties. Box 1 contains specific calculations to follow when generating an engineered hydrogel formulation ( $G: 100 \mathrm{~Pa} ; 4.0 \%$ (wt/vol), 20-kDa PEG-4MAL; $2.0 \mathrm{mM}$ RGD; GPQ-W cross-linker) that supports the in vitro generation of HIOs from hPSC-derived spheroids and the culture of HLOs ${ }^{16}$. This engineered formulation should be used as a starting point for designing a PEG-4MAL hydrogel for the in vitro generation and culture of the $\mathrm{HO}$ of interest. Further variations of the biophysical and biochemical matrix properties described above can be explored if the initial hydrogel formulation (Box 1) does not support the viability and growth of the human spheroid/HO of interest. Further discussion regarding how to vary the biophysical and biochemical matrix properties is presented in Box 1.

\section{Murine model for HIO transplant into colonic wounds}

The Procedure also explains how to deliver hydrogel-containing HIOs generated in the PEG-4MAL hydrogel or a biologically derived matrix to colonic mucosal wounds by injection via a colonoscope. This has previously been shown to result in organoid survival, engraftment, and accelerated wound repair ${ }^{16}$. Briefly, a miniaturized colonoscope system equipped with biopsy forceps is used to biopsy-injure the colonic mucosa of mice (as previously described ${ }^{24,25}$ ) and to inject an HO-containing hydrogel $1 \mathrm{~d}$ after wounding with the aid of a custom-made injection device (Fig. 4). 
To enable delivery via a small-bore device, liquid precursors of the hydrogel are administered through the colonoscope, and the polymerized hydrogel forms in situ at the injection site. We recommend using NOD-scid IL2Rg-null (NSG) mice as recipients for transplantation of human organoids using synthetic hydrogels to minimize immune rejection by the host of the transplanted human tissue. NSG mice have been demonstrated to be consistently better for human stem cell engraftment, as compared with several other immunocompromised mouse strains ${ }^{26}$. We recommend using male animals $\sim 12$ weeks old, as male mice have longer colons than do females, providing more space for colonoscopy purposes. At 12 weeks, mice are less susceptible to intestinal perforations as a consequence of the biopsy injury and have optimal repair programming. Nevertheless, we have conducted studies with C57BL/B6 mice at different ages and found no differences in wound repair between 3-month-old and 1-year-old mice. We have previously used 8-week-old, male NSG mice as a proof of concept for the use of the synthetic hydrogel as a delivery vehicle for HIOs to murine colonic wounds using a colonoscope, promoting HIO engraftment and accelerated wound healing ${ }^{16}$.

The ability of this synthetic matrix to deliver HOs via endoscopic techniques and the minimal toxicity and inflammatory response associated with its use in vivo ${ }^{18,20}$ are a proof of concept that hydrogel-encapsulated HOs can be used therapeutically to treat intestinal injury, overcoming the limitations associated with the use of Matrigel for $\mathrm{HO}$ technologies ${ }^{16}$. Moreover, the ability to tune the polymerization kinetics (gelling rate) of this injectable system renders possible its application in other settings. Adjusting the $\mathrm{pH}$ of the hydrogel precursor solutions to slightly below physiological $\mathrm{pH}(6.5-7.4)$ reduces the reaction kinetics. Reducing the speed of the reaction can help prevent premature crosslinking, which may occur during hydrogel delivery via custom-made devices such as the injection device we use in the Procedure (Fig. 4). By controlling the polymerization rate, different physical forms of the hydrogel can be generated.

\section{Alternative possible delivery methods and animal models that could be used}

We hypothesize that our system could be used in a variety of different ways to administer HOs in vivo, as we have shown the potential of PEG-4MAL hydrogels as a dynamic delivery vehicle for different cellular and noncellular systems via distinct delivery methods to a variety of in vivo sites, for example, as a patch that forms on the surface of the heart to localize mesenchymal stem cells in rats ${ }^{27}$, as an injectable matrix to deliver vasculogenic proteins that promote the vascularization and engraftment of pancreatic islets in rodents ${ }^{21}$, or as a liquid drug carrier delivered via a double-lumen catheter to produce a conformal drug depot in the pericardial space of pigs $^{28}$. Such strategies establish the potential of PEG-4MAL hydrogels as a dynamic organoid delivery vehicle that can be adapted to distinct delivery sites and animal models.

\section{Materials}

\section{Biological materials}

- Human spheroid or organoid of choice derived from hESCs or hiPSCs. We have successfully used human intestinal spheroids or HIOs derived as described in 
refs. ${ }^{1,2}$ from hPSC H9 (NIH registry no. 0062) and hiPSC line 20.1 (generated by the Pluripotent Stem Cell Facility, Cincinnati Children's Hospital Medical Center ${ }^{1}$ ). We have also successfully used HLOs derived as described in ref. ${ }^{5}$ from hESCs (UM63-1; NIH registry no. 0277) ! CAUTION We regularly monitor stem cell lines for chromosomal karyotype. For this purpose, the number of chromosomes is confirmed, chromosomal abnormalities are ruled out, and sex chromosomes of each line are confirmed. An assessment of functional and molecular authentication for pluripotency and for the ability of the cells to undergo multilineage differentiation is performed using a panel of antibody and qRT-PCR markers.

- Recipient mice for transplant of organoids. We recommend using 12-week-old, male NOD-scid IL2Rg-null (NSG) mice for the example application we describe in the Procedure (Jackson Laboratory, stock no. 005557) ! CAUTION All animal studies must be reviewed and approved by the relevant animal care committees and must conform to all relevant national and institutional ethics regulations. The method we describe here was approved by the University of Michigan's Institutional Animal Care and Use Committee in accordance with US Department of Agriculture Animal and Plant Health Inspection Service regulations and the National Institutes of Health (NIH) Office of Laboratory Animal Welfare regulations governing the use of vertebrate animals.

\section{Reagents}

- $\quad$ PEG-4MAL macromer (MW 22,000, >95\% MAL functionalization; Laysan Bio, cat. no. 4arm-PEG-MAL-20K)

- $\quad$ RGD: GRGDSPC (AAPPTec, custom synthesis, purity:>95\%, trifluoroacetic acid (TFA) removal)

- GPQ-W: GCRDGPQGIWGQDRCG (AAPPTec, custom synthesis, purity:>95\%, TFA removal)

- $\quad$ Advanced DMEM-F12 medium (Invitrogen, cat. no. 12634-010)

- $\quad$ L-Glutamine (100x; Invitrogen, cat. no. 25030-081)

- Penicillin-streptomycin (100x; Invitrogen, cat. no. 15140-122)

- $\quad$ B27 supplement (50×; Invitrogen, cat. no. 17504044)

- $\quad$ N-2 supplement (100×; Gibco, cat. no. 17502048)

- $\quad$ Fetal bovine serum (FBS; Life Technologies, cat. no. 16000-044)

- $\quad$ Noggin (R\&D Systems, cat. no. 6057-NG)

- $\quad$ R-spondin1 (R\&D Systems, cat. no. 4645-RS)

- $\quad$ Epidermal growth factor (EGF; R\&D Systems, cat. no. 236-EG)

- $\quad$ Fibroblast growth factor 10 (FGF-10; R\&D Systems, cat. no. 345-FG-025/CF)

- $\quad$ HEPES solution (Sigma, cat. no. H0887)

Nat Protoc. Author manuscript; available in PMC 2020 May 21. 
- $\quad$ DPBS (Thermo Fisher Scientific, cat. no. 14040133)

- $\quad$ Matrigel (BD Biosciences, cat. no. 354234)

- $\quad$ Ketamine-HCL (Hospira, cat. no. RL-3760)

- $\quad$ Xylazine (Lloyd Laboratories, cat. no. 4811)

- $\quad \mathrm{NaOH}$

\section{Equipment}

- $\quad$ Stereomicroscope (Olympus, model no. SZ61)

- Horizontal clean bench (Labcono)

- Costar multiwell, ultra-low-attachment plates (Corning, cat. no. 3473; 24-well plates are recommended)

- $\quad$ Costar Spin-X centrifuge tubes (Cole-Palmer, cat. no. UX-01937-32)

- $\quad$ Microcentrifuge tubes (VWR, cat. no. 10025-724)

- $\quad$ High-precision analytical balance (Mettler Toledo, cat. no. XPE105, or equivalent)

- $\quad$ pH bench meter (Mettler Toledo, cat. no. SC S220-B)

- $\quad$ MI-410 combination $\mathrm{pH}$ microprobe (Microelectrodes, or equivalent)

- $\quad$ Microcentrifuge (Beckman Coulter, cat. no. B30137)

- $\quad$ Large-orifice pipette tips (USA Scientific, cat. nos. 1011-8410 and 1011-9410)

- Forceps and/or tungsten needle

- $\quad$ Disposable scalpel (size 15; Exelint, cat. no. 29556)

- $\quad$ Colonoscope (Mainz Coloview Veterinary Endoscope; Karl Storz)

- $\quad$ Biopsy forceps (Mainz Coloview Veterinary Endoscope; Karl Storz)

- $\quad$ 27-gauge needles (BD, cat. no. 305109)

- $\quad$ TB syringes (BD, cat. no. 309659)

- $\quad$ Polyethylene tubing (Becton Dickinson, cat. no. 427406)

- $\quad$ Plastic feeding (gavage) needles (Instech, cat. no. GTP-20-30-50)

- $\quad$ 10-mL syringes (BD, cat. no. 309604)

\section{Reagent setup}

20 mM HEPES buffer (pH 7.4)-To prepare $100 \mathrm{~mL}$ of $20 \mathrm{mM}$ HEPES buffer, mix $2 \mathrm{~mL}$ of HEPES solution with $90 \mathrm{~mL}$ of DPBS. Adjust the $\mathrm{pH}$ to 7.4 using $6 \mathrm{M} \mathrm{NaOH}$ and bring the final volume of the resulting solution to $100 \mathrm{~mL}$ with DPBS. Sterile $20 \mathrm{mM}$ HEPES buffer can be stored for 2 months at room temperature $\left(\sim 20^{\circ} \mathrm{C}\right)$. 
Intestine growth medium-For $500 \mathrm{~mL}$ of intestine growth medium, pool together 459.7 $\mathrm{mL}$ of advanced DMEM-F12 medium, $5 \mathrm{~mL}$ of L-glutamine (final concentration $=2 \mathrm{mM}$ ), $7.5 \mathrm{~mL}$ of HEPES solution (final concentration $=15 \mathrm{mM}$ ), $20 \mathrm{~mL}$ of B27 supplement $(1 \times$ final dilution $=2 \mathrm{~mL}$ per $50 \mathrm{~mL}$ of medium), $5 \mathrm{~mL}$ of penicillin-streptomycin (final concentration $=100 \mathrm{U} / \mathrm{mL}$ penicillin, $100 \mu \mathrm{g} / \mathrm{mL}$ streptomycin), $200 \mu \mathrm{L}$ of Noggin (250 $\mu \mathrm{g} / \mathrm{mL}$; final concentration $=100 \mathrm{ng} / \mathrm{mL}), 100 \mu \mathrm{L}$ of EGF $(500 \mu \mathrm{g} / \mathrm{mL}$; final concentration $=$ $100 \mathrm{ng} / \mathrm{mL})$, and $2.5 \mathrm{~mL}$ of R-spondin $1(100 \mu \mathrm{g} / \mathrm{mL}$; final concentration $=500 \mathrm{ng} / \mathrm{mL})$. The intestine growth medium is best if freshly made but can be stored for 1 week at $4{ }^{\circ} \mathrm{C}$.

Foregut basal medium-For $500 \mathrm{~mL}$ of foregut basal medium, pool together $470 \mathrm{~mL}$ of advanced DMEM-F12 medium, $5 \mathrm{~mL}$ of L-glutamine (final concentration $=2 \mathrm{mM}$ ), $5 \mathrm{~mL}$ of HEPES solution (final concentration $=10 \mathrm{mM}$ ), $5 \mathrm{~mL}$ of penicillin-streptomycin (final concentration $=100 \mathrm{U} / \mathrm{mL}$ penicillin, $100 \mu \mathrm{g} / \mathrm{mL}$ streptomycin), $10 \mathrm{~mL}$ of B27 supplement ( $1 \times$ final dilution $=1 \mathrm{~mL}$ per $50 \mathrm{~mL}$ of medium), and $5 \mathrm{~mL}$ of $\mathrm{N}-2$ supplement $(1 \times$ final dilution $=0.5 \mathrm{~mL}$ per $50 \mathrm{~mL}$ of medium). Foregut basal medium can be stored at $4{ }^{\circ} \mathrm{C}$ for up to 1 month.

HLO growth medium-On the day of use, add $49.5 \mathrm{~mL}$ of foregut basal medium to a 50$\mathrm{mL}$ conical tube. Add $500 \mu \mathrm{L}$ of FBS (final concentration $=1 \%)$ and $250 \mu \mathrm{L}$ of FGF-10 (100 $\mu \mathrm{g} / \mathrm{mL}$ stock; final concentration $=500 \mathrm{ng} / \mathrm{mL}$ ). The $\mathrm{HLO}$ growth medium is best if freshly made but can be stored for 1 week at $4{ }^{\circ} \mathrm{C}$.

Anesthesia solution-Prepare anesthesia solution comprising ketamine $(100 \mathrm{mg} / \mathrm{kg}$ of murine body weight) and xylazine (10 mg/kg of murine body weight) in DPBS. $5 \mathrm{~mL}$ of anesthesia solution is needed per $1 \mathrm{~kg}$ of murine body weight; prepare an appropriate volume. Prepare this solution fresh each time.

Hydrogel components-Prepare aliquots of each hydrogel component. We prepare PEG-4MAL macromer, RGD, and GPQ-W peptides (powder) in 100-mg, 25-mg, and 50-mg aliquots, respectively, to avoid humidity exposure caused by the components experiencing multiple temperature changes. We also recommend dissolving the necessary amounts of hydrogel components on the same day of experimentation. We do not recommend dissolving and then storing the PEG-4MAL macromer, as the maleimide groups may hydrolyze.

CRITICAL Upon receipt, immediately store PEG-4MAL macromer, RGD, and GPQ-W peptides (powder) at $-20{ }^{\circ} \mathrm{C}$.

\section{Equipment setup}

Custom-made device for injections-Remove the metallic part of a 27-gauge needle (o.d. $=0.41 \mathrm{~mm}$ ) from its hub. Carefully attach the blunt end of the needle to one end of a $10-\mathrm{cm}$ piece of intramedic polyethylene tubing (o.d. $=1.09 \mathrm{~mm}$ ), leaving the needle bevel exposed. Connect another complete needle to the other end of the tubing through the needle bevel (Fig. 4). Prepare one custom-made device for each injection to avoid clogging. We recommend preparing the custom-made device before starting the experimental procedure described in Step 13B. 


\section{Procedure}

\section{Preparation of hydrogel precursor solutions Timing 1-2 $\mathrm{h}$}

1. Allow one aliquot each of PEG-4MAL macromer, adhesive peptide (RGD), and cross-linker (GPQ-W) to reach room temperature.

2. Weigh out $100.0 \mathrm{mg}$ of PEG-4MAL macromer, $4.06 \mathrm{mg}$ of RGD, and $13.27 \mathrm{mg}$ of GPQ-W, using a high-precision analytical balance, and place each component into a separate microcentrifuge tube (see Box 1 for sample calculation).

$\triangle$ CRITICAL STEP This amount of hydrogel precursors produces a total 2.5$\mathrm{mL}$ hydrogel volume of $4.0 \%$ (wt/vol), 20-kDa PEG-4MAL hydrogels $(G=100$ $\mathrm{Pa}$ ) functionalized with $2.0 \mathrm{mM}$ RGD and cross-linked with GPQ-W peptide.

This specific hydrogel formulation was previously reported for HIO generation and HLO culture ${ }^{16}$, and thus serves as a starting point to identify a hydrogel formulation for the generation and culture of the $\mathrm{HO}$ of interest.

$\triangle$ CRITICAL STEP Variations of the biophysical and biochemical matrix properties can be explored if the hydrogel formulation described here does not support the viability and growth of the human spheroid/HO of interest. These changes may change the values used in this example but do not change the steps described in this protocol. Refer to Box 1 for further guidance.

3. Dissolve the GPQ-W and RGD peptides using $0.5 \mathrm{~mL}$ of $20 \mathrm{mM}$ HEPES buffer for each peptide to produce $15.57 \mathrm{mM}$ and $11.76 \mathrm{mM}$ peptide solutions, respectively, after considering the purity of the peptides (as demonstrated in Box $1)$.

$\triangle$ CRITICAL STEP Adjust the $\mathrm{pH}$ of each peptide solution to 7.4 using $6 \mathrm{M}$ $\mathrm{NaOH}$, measuring $\mathrm{pH}$ using a $\mathrm{pH}$ meter combined with a $\mathrm{pH}$ microprobe.

$\triangle$ CRITICAL STEP These RGD and GPQ-W peptide concentrations correspond to five times the concentration of their final density (see Box 1 for example calculation). This concentration factor can be changed, given that all maleimide groups of the PEG-4MAL macromer are conjugated after all hydrogel components are mixed.

4. Filter the RGD solution (prepared in Step 3), GPQ-W solution (prepared in Step 3 ), and $1 \mathrm{~mL}$ of $20 \mathrm{mM}$ HEPES buffer by transferring each solution to a separate Costar Spin-X centrifuge tube and centrifuging each tube at 9,000 $g$ for $1 \mathrm{~min}$ at room temperature.

5. Dissolve the PEG-4MAL macromer using $1 \mathrm{~mL}$ of $20 \mathrm{mM}$ HEPES buffer, filtered in Step 4, to produce a $4.55 \mathrm{mM}$ solution (Fig. 1).

$\triangle$ CRITICAL STEP This PEG-4MAL macromer concentration corresponds to 2.5 times the concentration of its final density (see Box 1 for example calculation). This concentration factor can be changed, given that all maleimide groups of the PEG-4MAL macromer are conjugated after all hydrogel components are mixed (see Box 1 for example calculation). 
$\triangle$ CRITICAL STEP Steps 5-13 must be performed in a sterile environment (e.g., using a horizontal clean bench and sterile tools).

6. Mix the solutions of PEG-4MAL macromer and adhesive peptide (RGD) in a 2:1 PEG-4MAL/adhesive peptide volume ratio (1.0 mL of PEG-4MAL macromer and $0.5 \mathrm{~mL}$ of RGD peptide, for this example) to generate the solution of the functionalized PEG-4MAL precursor. Then incubate the solution thus obtained for at least $15 \mathrm{~min}$ at $37^{\circ} \mathrm{C}$ (Fig. 1).

$\triangle$ CRITICAL STEP This step produces the conjugation between the maleimide groups of the PEG-4MAL macromer and the thiol groups of the peptides.

$\triangle$ CRITICAL STEP The solution can then be stored at room temperature for no more than $3 \mathrm{~h}$, in order to avoid hydrolyzation of the maleimide groups and to allow time to complete the next step.

\section{Preparation of suspensions}

7. Prepare an hPSC-derived 3D spheroid (option A) or an hPSC-derived HO (option B) suspension in a separate microcentrifuge tube.

$\triangle$ CRITICAL Option A can be applied to any type of hPSC-derived 3D spheroid generated in vitro by directed differentiation protocols (e.g., midgut and hindgut spheroids for the generation of HIOs as previously described ${ }^{1,2}$ ) for the purpose of generating HOs. Option B can be applied to any type of hPSCderived $\mathrm{HO}$ that was generated in Matrigel or a similar matrix for the period of time required by its differentiation protocol (e.g., HIOs and HLOs generated in Matrigel for at least $14 \mathrm{~d}$, as described in refs. ${ }^{1,2,5,16}$ ).

\section{A. Preparation of a human spheroid suspension - Timing $30 \mathrm{~min}$}

i. Harvest floating hiPSC- or hESC-derived spheroids that have reached the desired differentiation stage (e.g., midgut and hindgut spheroids generated in cultures on day 3 and day 4 of $\mathrm{mid} /$ hindgut induction, as previously described in Steps 1-19 of ref. ${ }^{2}$ ) by pipetting with large-orifice pipette tips, and transfer them to a microcentrifuge tube containing intestine growth medium (Fig. 2a).

ii. Adjust the volume of the spheroid suspension using growth medium (to a total volume of at least $0.5 \mathrm{~mL}$, for this example) to obtain a spheroid density that corresponds to five times the concentration of their final density in the hydrogels, and keep the suspension on ice (Fig. 2a). We recommend using a final density of 20-30 spheroids per $40 \mu \mathrm{L}$ of hydrogel and storing the spheroid solution on ice for no longer than $1 \mathrm{~h}$, as previously reported for intestinal spheroids ${ }^{16}$.

B. Preparation of a human organoid suspension $\bullet$ Timing $30 \mathrm{~min}$ 
i. Mechanically dislodge HOs generated in Matrigel (e.g., HIOs and HLOs, as previously described in Step 33 of ref. ${ }^{2}$ ) or a similar matrix by vigorously pipetting the matrix with largeorifice pipette tips to free the organoids, and then transfer them to a microcentrifuge tube containing intestine or HLO growth medium.

A CRITICAL STEP We have used HIOs and HLOs that have been generated from Matrigel-encapsulated spheroids for at least $14 \mathrm{~d}$ (as previously reported ${ }^{16}$ ), although it is probable that other types of hPSC-derived HOs that were generated in Matrigel or a similar matrix for a different time frame can be used.

ii. Adjust the volume of the $\mathrm{HO}$ suspension using growth medium (for this example, to a total volume of at least 0.5 $\mathrm{mL}$ ) to obtain an $\mathrm{HO}$ density that corresponds to five times the concentration of their final density in the hydrogels, and keep the suspension on ice. We recommend using a final density of two to four HOs per $40 \mu \mathrm{L}$ of hydrogel and storing the $\mathrm{HO}$ solution on ice for no longer than $1 \mathrm{~h}$, as previously reported for HIOs and HLOs ${ }^{16}$.

\section{Casting of synthetic hydrogel Timing $1 \mathrm{~h}$}

8. Mix the human spheroid suspension or the $\mathrm{HO}$ suspension with a functionalized PEG-4MAL precursor solution (prepared in Step 6) at a 3:1 functionalized PEG-4MAL/cell suspension volume ratio (for this example, $1.5 \mathrm{~mL}$ of functionalized PEG-4MAL precursor and $0.5 \mathrm{~mL}$ of human spheroid suspension or HO suspension), using large-orifice pipette tips, and keep the suspension on ice for no longer than $30 \mathrm{~min}$ (Fig. 2a).

9. Add the cross-linking peptide solution (GPQ-W) prepared in Step 4 (20\% of the desired final hydrogel volume, as shown in Box 1 -we recommend adding $8 \mu \mathrm{L}$ of GPQ-W to each well, to produce $40 \mu \mathrm{L}$ of hydrogel in each well) to the bottom (centered) of each well of a multiwell plate (Fig. 2b).

$\triangle$ CRITICAL STEP Perform this step in the shortest time possible, in order to avoid cross-linker evaporation.

10. Pipette the mixture comprising the functionalized PEG-4MAL precursor and the cell mixture prepared in Step 8 ( $80 \%$ of the desired final hydrogel volume, as shown in Box 1-as mentioned in the previous step, we recommend adding 32 $\mu \mathrm{L}$ of this solution, to produce $40 \mu \mathrm{L}$ of hydrogel in each well) into the crosslinker solution in each well, using large-orifice pipette tips (Fig. 2b).

$\triangle$ CRITICAL STEP Mix the solution by pipetting several times to obtain a homogeneous solution.

$\triangle$ CRITICAL STEP Use a new pipette tip to cast each different hydrogel. 
$\triangle$ CRITICAL STEP Perform this step in the shortest time possible, in order to avoid cross-linker evaporation.

11. Allow the hydrogel to form by incubating the plate at $37^{\circ} \mathrm{C}$ for $20 \mathrm{~min}$.

\section{? TROUBLESHOOTING}

12. Overlay each hydrogel with $500 \mu \mathrm{L}$ of medium (e.g., intestine growth medium for intestinal spheroids or HIOs, or HLO growth medium for HLOs), ensuring that the hydrogel is covered by medium, and then incubate under their corresponding culture conditions (e.g., in a $5 \% \mathrm{CO}_{2}$ incubator at $37{ }^{\circ} \mathrm{C}$ for culture of HIOs, as previously described ${ }^{16}$ ). Replace the medium every $4 \mathrm{~d}$, or when the phenol red (included in the advanced DMEM-F12 medium) in the medium turns yellow (Fig. 2b).

\section{Organoid generation/culture and delivery}

13. Passage human spheroids or HOs encapsulated in PEG-4MAL hydrogels every 7-10 d (option A), and continue the culture for the desired time frame. If generating HIOs or culturing HIOs generated in a different matrix, these can then be delivered with synthetic hydrogel precursor solutions via injection into a colonic wound (option B).

$\triangle$ CRITICAL The hydrogel degradation, and thus the frequency of passages, may vary according to the type and density of encapsulated spheroids/HOs within the hydrogel. Proceed to passage human spheroids/HOs when these start to settle to the bottom of the well of the culture plate.

$\triangle$ CRITICAL The culture time frame for human spheroids can vary according to their type and their time line for expansion into HOs. For reference, we allow at least $14 \mathrm{~d}$ of expansion of encapsulated intestinal spheroids into HIOs (Fig. $2 b$ ), and we have successfully passaged HIOs for up to three passages, spanning $\sim 21 \mathrm{~d}$, stopping only for an experimental endpoint, as previously reported ${ }^{16}$. It is likely that HIOs will continue to grow and expand for a much longer period than the mentioned $21 \mathrm{~d}$.

A. Organoid passaging Timing 5 min per well containing $\sim 5 \mathrm{HOs}$

$\triangle$ CRITICAL Organoid passaging as described here is intended for hPSC-derived HOs and differs from the trypsin-based passage protocols used for primary tissue-derived organoids.

i. Repeat Steps 1-6 ('Preparation of hydrogel precursor solutions') to prepare the hydrogel precursor solutions needed to make new hydrogels.

ii. Mechanically dislodge the organoids from the PEG-4MAL hydrogel by vigorously pipetting the hydrogel with largeorifice pipette tips to free the organoids from the matrix. 
iii. Pool all the hydrogels + organoids + medium from each well in a sterile Petri dish containing $10 \mathrm{~mL}$ of warm advanced DMEM-F12 medium. By using a sterile tungsten needle or sterile fine forceps, dislodge any large pieces of hydrogel that still adhere to the organoids.

iv. Manually cut the organoids into halves using a scalpel under a stereomicroscope.

$\triangle$ CRITICAL STEP This step is required only for HOs that have been previously reported to require manual cutting during passaging. For example, manual cutting is required for HIOs (as previously described in ref. ${ }^{2}$ ) because of the mesenchymal layer present and large size. For other types of HOs that do not require manual cutting, such as $\mathrm{HLOs}^{5}$, this step can be skipped.

v. Transfer the organoids to a microcentrifuge tube, and adjust the volume of the suspension using growth medium (for this example, to a total volume of at least $0.5 \mathrm{~mL}$ ) to obtain an $\mathrm{HO}$ density that corresponds to five times the concentration of their final density in the hydrogels, and keep the suspension on ice. We recommend using a final density of two to four HOs per $40 \mu \mathrm{L}$ of hydrogel and storing the $\mathrm{HO}$ solution on ice for no longer than $1 \mathrm{~h}$, as previously reported for HIOs and HLOs ${ }^{16}$.

vi. Repeat Steps 8-13 ('Synthetic hydrogel casting'), using the organoid suspension prepared in Step 13A(v), to form new HO-containing hydrogels.

vii. Culture HOs under their corresponding culture conditions (e.g., in a $5 \% \mathrm{CO}_{2}$ incubator at $37^{\circ} \mathrm{C}$ for culture of $\mathrm{HIOs}$, as previously described ${ }^{16}$ ).

\section{? TROUBLESHOOTING}

B. Organoid encapsulation and injection into colonic mucosal wound bed $\bullet$ Timing $5 \mathrm{~h}$

A CRITICAL Organoid encapsulation and injection into colonic mucosal wound beds as described here is recommended for hPSCderived HIOs generated in PEG-4MAL hydrogel or Matrigel, as previously demonstrated ${ }^{16}$.

i. One day before injection of HOs, mechanically induce mucosal wounds in recipient mice. Briefly, anesthetize the mice by intraperitoneal injection of the anesthesia solution. Ensure that the mouse is adequately anesthetized by testing its pedal reflex and monitoring its respiration $5 \mathrm{~min}$ post 
injection of anesthesia. We use NSG mice and use the biopsy forceps included in the colonoscope system to induce injury. Lubricate the anus with a drop of PBS, and insert the colonoscope probe until you reach the flexure. Introduce the biopsy forceps through the auxiliary pocket until the tip of the forceps is observed through the high-resolution colonoscope camera. Open and close the forceps to pinch the colonic mucosa, and swiftly pull the biopsy forceps to make a wound of $\sim 1 \mathrm{~mm}$ in diameter in the colonic mucosa of the mouse. We recommend making four to five wounds in each mouse, as previously reported ${ }^{16,25}$. The process takes from 2 to $5 \mathrm{~min}$ per mouse, depending on handler expertise. Further details on induction of mucosal wounds can be found in ref. ${ }^{25}$.

! CAUTION All animal studies must be reviewed and approved by the relevant animal care committees and must conform to all relevant national and institutional ethics regulations.

ii. Repeat Steps 1-6 ('Preparation of hydrogel precursor solutions') to prepare the hydrogel precursor solutions needed to make new 4\% (wt/vol) PEG-4MAL hydrogels functionalized with $2.0 \mathrm{mM}$ RGD and cross-linked with GPQW peptide for the injections (Fig. 1; Box 1). We have previously reported that this hydrogel formulation promotes HIO engraftment into the host tissue and accelerated wound repair $^{16}$.

iii. Mechanically dislodge HIOs generated within PEG-4MAL hydrogels (as described above) or Matrigel (as previously described $^{1,2,16}$ ) by vigorously pipetting with large-orifice pipette tips to free the organoids from the matrix (Fig. 4).

iv. Transfer the HIOs to a microcentrifuge tube, and adjust the volume of the suspension, using intestine growth medium (for this example, to a total volume of at least $0.5 \mathrm{~mL}$ ) to obtain an HIO density that corresponds to five times the concentration of their final density in the hydrogels, and keep the suspension on ice. We recommend using a final density of ten HIOs per $50 \mu \mathrm{L}$ of hydrogel and storing the HIO solution on ice for no longer than $1 \mathrm{~h}$, as previously reported ${ }^{16}$ (Fig. 4).

v. Load $10 \mu \mathrm{L}$ of the cross-linker solution into the custom-made device prepared as described in the 'Equipment setup' section.

$\triangle$ CRITICAL STEP One custom-made device is needed for each injection to avoid clogging. 
vi. Mix the HIO suspension prepared in Step 13B(iv) with the PEG-4MAL-RGD precursor solution prepared in Step 13B(ii) in a 3:1 PEG-4MAL-RGD/HIO suspension volume ratio (for this example, $1.5 \mathrm{~mL}$ of functionalized PEG-4MAL precursor and $0.5 \mathrm{~mL}$ of HIO suspension), using large-orifice pipette tips, and keep the resulting mixture on ice for the duration of the following steps (Fig. 4).

vii. Load the PEG-4MAL-RGD precursor + HIO mixture solution prepared in the previous step into a 1-mL TB syringe. Note that $40 \mu \mathrm{L}$ of this solution will be used per injection (Fig. 4).

viii. Connect the custom-made device containing the cross-linker solution (Step 13B(v)) to the TB syringe containing the PEG-4MAL-RGD + HIO suspension (Fig. 4). Insert the needle bevel through the colonoscope forceps auxiliary pocket.

ix. Anesthetize the mouse by intraperitoneal injection of the anesthesia solution. Ensure that the mouse is adequately anesthetized by testing its pedal reflex and monitoring its respiration $5 \mathrm{~min}$ post injection of anesthesia.

x. Use DPBS in a plastic gavage needle to lubricate and insert the colonoscope probe into the rectum. Use a colonoscope equipped with a high-resolution camera to identify the mucosal wounds.

xi. Once a wound has been found, position the colonoscope probe close to the distal end of the wound. Push the tubing through the auxiliary pocket until the needle bevel is observed through the camera, and proceed to carefully penetrate the mucosa and locate the needle bevel right at the wound bed (as demonstrated previously ${ }^{25}$; Fig. 4, Fig. 5, and Supplementary Video 1). To assess the effect of $\mathrm{HO}$ delivery into the wounds, we recommend taking videos or pictures of the wounds with the colonoscope's camera right before the injection to enable the percentage of wound healing to be determined.

\section{? TROUBLESHOOTING}

xii. Inject $40 \mu \mathrm{L}$ of the functionalized PEG-4MAL-RGD + HIO suspension, which will mix with the cross-linker solution in the tubing, leading to the in situ formation of the hydrogel at the injection site. Monitor the success of the procedure by checking to see that a small, yet visible, protrusion appears at the injection site (Supplementary Video 1 and as demonstrated previously ${ }^{25}$ ).

? TROUBLESHOOTING 
xiii. Pull the needle out and repeat the injection at a different wound site if required.

$\triangle$ CRITICAL STEP We recommend performing one injection per wound to lessen the risk of perforation.

A CRITICAL STEP A new custom-made device containing the cross-linker will be needed for each injection. Keep the anesthetized mouse on a heated pad to avoid hypothermia and monitor until it is awake (usually takes $1 \mathrm{~h}$ after injection of anesthesia).

$\triangle$ CRITICAL STEP To assess the effect of HO delivery in the wounds, we recommend taking videos or pictures of the wounds with the colonoscope's camera right before the injection and $4 \mathrm{~d}$ after the injections to calculate the percentage of wound healing, as previously described ${ }^{16,25}$.

A CRITICAL STEP To evaluate HO engraftment, different techniques (e.g., in situ hybridization, immunofluorescence, western blot, and flow cytometry) can be used to detect specific markers of human tissue on the mouse colonic tissue. We have previously evaluated $\mathrm{HO}$ engraftment into mouse colonic wounds using immunofluorescence (Fig. 6) and in situ hybridization ${ }^{16}$.

\section{Troubleshooting}

Troubleshooting advice can be found in Table 1.

\section{Timing}

Steps 1-6, preparation of hydrogel precursor solutions: $1-2 \mathrm{~h}$

Step 7A, preparation of human spheroid suspension: $30 \mathrm{~min}$

Step 7B, preparation of human organoid suspension: $30 \mathrm{~min}$

Steps $8-12$, casting of synthetic hydrogel: $1 \mathrm{~h}$

Step 13A, organoid passaging: 5 min per well containing $~ 5 \mathrm{HOs}$

Step 13B, organoid encapsulation and injection into colonic mucosal wound bed: $5 \mathrm{~h}$

\section{Anticipated results}

This protocol outlines the synthesis of PEG-4MAL hydrogels that can generate HOs from hPSC-derived 3D spheroids and maintain the HOs for multiple passages without the need for Matrigel encapsulation. Intestinal spheroids embedded in the engineered hydrogel ( $G=$ $100 \mathrm{~Pa} ; 4.0 \%$ (wt/vol), 20-kDa PEG-4MAL; 2.0 mM RGD; and GPQ-W cross-linker) 
develop into HIOs with lumens after 4-5 d in culture (Fig. 7a,b). During expansion, spheroids change shape and display epithelial budding at the interface with the hydrogel (Fig. 7b). Furthermore, hydrogel-generated HIOs can be passaged into fresh hydrogels every 7-10 d to support further differentiation of HIOs, demonstrating that PEG-4MAL hydrogels can generate and maintain fully developed HIOs to levels similar to those of Matrigel (Fig. $7 \mathrm{c}, \mathrm{d})$. We have successfully passaged HIOs for up to three passages, spanning $21 \mathrm{~d}$, stopping only for an experimental endpoint. It is probable that HIOs will continue to grow and expand for a much longer period than the mentioned $21 \mathrm{~d}$. Established HIOs grow in size, change shape, maintain a central lumen, and display cell outgrowths migrating into the hydrogel (Fig. 7b,c).

In addition, this synthetic matrix can be used as a delivery vehicle to promote HIO engraftment and accelerated wound healing of injured colonic tissue ${ }^{16}$. In Fig. 7, we show the results obtained during our previous study ${ }^{16}$, demonstrating that cells expressing markers of human cells have engrafted into the host tissue 4 weeks after in vivo injection of HIOcontaining PEG-4MAL precursor solutions into the colonic mucosal wound bed. Because we have assessed $\mathrm{HIO}$ engraftment only at 4 weeks post injection, we do not know the specific time line of HIO engraftment or whether engraftment can be assessed at an earlier time point.

\section{Reporting Summary}

Further information on experimental design is available in the Nature Research Reporting Summary linked to this article.

\section{Supplementary Material}

Refer to Web version on PubMed Central for supplementary material.

\section{Acknowledgements}

This research was supported by the National Institutes of Health (A.J.G. was supported by R01 AR062368 and R01 AR062920; A.N. was supported by R01 DK055679, R01 DK059888, DK055679, and DK089763) and a seed grant from the Regenerative Engineering and Medicine Research Center between Emory University, Georgia Tech and the University of Georgia. J.R.S. was supported by the Intestinal Stem Cell Consortium (U01DK103141), a collaborative research project funded by the National Institute of Diabetes and Digestive and Kidney Diseases (NIDDK) and the National Institute of Allergy and Infectious Diseases (NIAID), the NIAID Novel, Alternative Model Systems for Enteric Diseases (NAMSED) consortium (U19AI116482), and PHS grant UL1TR000454 from the Clinical and Translational Science Award Program. R.C.-A. was supported by a National Science Foundation Graduate Research Fellowship (DGE-1650044) and the Alfred P. Sloan Foundation's Minority PhD (MPHD) Program (G-2016-20166039). M.Q. was supported by a fellowship from the Crohn's and Colitis Foundation of America (CCFA 326912). We thank A. Heinen for technical support.

\section{Reference}

1. Spence JR et al. Directed differentiation of human pluripotent stem cells into intestinal tissue in vitro. Nature 470, 105-109 (2011). [PubMed: 21151107]

2. McCracken KW, Howell JC, Wells JM \& Spence JR Generating human intestinal tissue from pluripotent stem cells in vitro. Nat. Protoc 6, 1920-1928 (2011). [PubMed: 22082986]

3. Fatehullah A, Tan SH \& Barker N Organoids as an in vitro model of human development and disease. Nat. Cell Biol 18, 246-254 (2016). [PubMed: 26911908] 
4. Clevers H Modeling development and disease with organoids. Cell 165, 1586-1597 (2016). [PubMed: 27315476]

5. Dye BR et al. In vitro generation of human pluripotent stem cell derived lung organoids. eLife 4, e05098 (2015).

6. Miller AJ et al. In vitro induction and in vivo engraftment of lung bud tip progenitor cells derived from human pluripotent stem cells. Stem Cell Rep. 10, 101-119 (2017).

7. Lancaster MA \& Knoblich JA Generation of cerebral organoids from human pluripotent stem cells. Nat. Protoc 9, 2329-2340 (2014). [PubMed: 25188634]

8. Nakano T et al. Self-formation of optic cups and storable stratified neural retina from human ESCs. Cell Stem Cell 10, 771-785 (2012). [PubMed: 22704518]

9. Takasato M, Er PX, Chiu HS \& Little MH Generation of kidney organoids from human pluripotent stem cells. Nat. Protoc 11, 1681 (2016). [PubMed: 27560173]

10. Wells JM \& Spence JR How to make an intestine. Development 141, 752-760 (2014). [PubMed: 24496613]

11. Hughes CS, Postovit LM \& Lajoie GA Matrigel: a complex protein mixture required for optimal growth of cell culture. Proteomics 10, 1886-1890 (2010). [PubMed: 20162561]

12. Broutier $\mathrm{L}$ et al. Culture and establishment of self-renewing human and mouse adult liver and pancreas 3D organoids and their genetic manipulation. Nat. Protoc 11, 1724 (2016). [PubMed: 27560176]

13. Miyoshi H \& Stappenbeck TS In vitro expansion and genetic modification of gastrointestinal stem cells in spheroid culture. Nat. Protoc 8, 2471 (2013). [PubMed: 24232249]

14. Cruz-Acuña R \& García AJ Synthetic hydrogels mimicking basement membrane matrices to promote cell-matrix interactions. Matrix Biol. 57-58, 324-333 (2016).

15. Gjorevski N et al. Designer matrices for intestinal stem cell and organoid culture. Nature 539, 560564 (2016). [PubMed: 27851739]

16. Cruz-Acuña R et al. Synthetic hydrogels for human intestinal organoid generation and colonic wound repair. Nat. Cell Biol 19, 1326-1335 (2017). [PubMed: 29058719]

17. Zhu J Bioactive modification of poly(ethylene glycol) hydrogels for tissue engineering. Biomaterials 31, 4639-4656 (2010). [PubMed: 20303169]

18. Phelps EA et al. Maleimide cross-linked bioactive PEG hydrogel exhibits improved reaction kinetics and cross-linking for cell encapsulation and in situ delivery. Adv. Mater 24, 64-70 (2012). [PubMed: 22174081]

19. Enemchukwu NO et al. Synthetic matrices reveal contributions of ECM biophysical and biochemical properties to epithelial morphogenesis. J. Cell Biol 212, 113-124 (2016). [PubMed: 26711502]

20. Phelps EA, Headen DM, Taylor WR, Thule PM \& Garcia AJ Vasculogenic bio-synthetic hydrogel for enhancement of pancreatic islet engraftment and function in type 1 diabetes. Biomaterials 34 , 4602-4611 (2013). [PubMed: 23541111]

21 . Weaver JD et al. Vasculogenic hydrogel enhances islet survival, engraftment, and function in leading extrahepatic sites. Sci. Adv 3, e1700184 (2017). [PubMed: 28630926]

22. Gjorevski N \& Lutolf MP Synthesis and characterization of well-defined hydrogel matrices and their application to intestinal stem cell and organoid culture. Nat. Protoc 12, 2263 (2017). [PubMed: 28981121]

23. Lu P, Takai K, Weaver VM \& Werb Z Extracellular matrix degradation and remodeling in development and disease. Cold Spring Harb. perspect. Biol. 3, a005058 (2011).

24. Leoni $\mathrm{G}$ et al. Annexin A1-containing extracellular vesicles and polymeric nanoparticles promote epithelial wound repair. J. Clin. Invest 125, 1215-1227 (2015). [PubMed: 25664854]

25. Bruckner $\mathrm{M}$ et al. Murine endoscopy for in vivo multimodal imaging of carcinogenesis and assessment of intestinal wound healing and inflammation. J. Vis. Exp, 10.3791/51875 (2014).

26. Greiner DL, Hesselton RA \& Shultz LD SCID mouse models of human stem cell engraftment. Stem Cells 16, 166-177 (1998). [PubMed: 9617892]

27. Levit RD et al. Cellular encapsulation enhances cardiac repair. J. Am. Heart Assoc 2, e000367 (2013). [PubMed: 24113327] 
28. Garcia JR et al. A minimally invasive, translational method to deliver hydrogels to the heart through the pericardial space. JACC: Basic Transl. Sci 2, 601-609 (2017). [PubMed: 30062173]

29. Noble JE \& Bailey MJA in Methods in Enzymology, Vol. 463 (eds. Burgess Richard R. \& Deutscher Murray P.) 73-95 (Academic Press, Cambridge, MA, 2009). [PubMed: 19892168] 


\section{Box 1 | Example calculation}

This is an example of calculations needed to prepare hydrogel precursor solutions starting with $100 \mathrm{mg}$ of PEG-4MAL macromer, which is used to produce a total $2.5-\mathrm{mL}$ hydrogel volume of $4.0 \%$ (wt/vol), 20-kDa PEG-4MAL hydrogels $(G: 100 \mathrm{~Pa}$ ) functionalized with $2.0 \mathrm{mM}$ RGD and cross-linked with GPQ-W peptide. This volume of PEG-4MAL hydrogel is enough for approximately sixty $40-\mu \mathrm{L}$ hydrogels for in vitro $\mathrm{HO}$ encapsulation or approximately fifty $50-\mu \mathrm{L}$ hydrogels for in vivo injections of HOcontaining hydrogels, as previously reported for HIO generation and HLO culture ${ }^{16}$. The total volume of hydrogels can be varied on the basis of the total volume and the number of hydrogels needed for experimentation. As discussed in the 'Experimental design' section, PEG-4MAL macromer size and density, and the type and density of adhesive and cross-linking peptides, can be varied—provided that all maleimide groups are conjugated - to reach the desired biophysical and biochemical matrix properties, respectively. Variations of the biophysical and biochemical matrix properties can be explored if the hydrogel formulation described here does not support the viability and growth of the human spheroid/HO of interest. In this case, this example would serve as a guide for the calculations required to form the desired hydrogel. Note that changes to the biophysical and biochemical matrix properties may change the values used in this example calculation but do not change the steps described in this protocol. As shown here, it is essential to consider the purity of the peptides and the maleimide substitution efficiency of the PEG-4MAL, provided by the vendors, to achieve complete cross-linking of the hydrogel. The volume fractions provided in the example ( 0.4 for PEG-4MAL and 0.2 for adhesive and cross-linking peptides) correspond to the volume ratios described in the protocol (2:1:1, PEG-4MAL/adhesive peptide/cross-linking peptide) and are recommended on the basis of previous reports ${ }^{16,19}$. It is recommended that this example of calculations be translated into a spreadsheet for convenience.

The specific hydrogel formulation provided in this example was previously reported for HIO generation and $\mathrm{HLO}$ culture ${ }^{16}$, and thus serves as a starting point for identifying a hydrogel formulation for the generation and culture of the $\mathrm{HO}$ of interest.

MW ${ }_{\text {PEG-4MAL }} 22,000 \mathrm{Da}$ (molecular weight of PEG-4MAL provided by the vendor)

$\mathrm{MW}_{\mathrm{RGD}}$ 690.6 $\mathrm{Da}$ (molecular weight of RGD provided by the vendor)

$\mathrm{MW}_{\mathrm{GPQ}-\mathrm{W}} 1704.9 \mathrm{Da}$ (molecular weight of GPQ-W provided by the vendor)

4 Maleimide groups per PEG-4MAL macromer

$P_{\text {PEG-4MAL }} 0.96$ (maleimide substitution efficiency provided by the vendor)

$P_{\text {RGD }} 0.85$ (RGD fraction concentration provided by the vendor)

$P_{\mathrm{GPQ}-\mathrm{W}} 0.8$ (GPQ-W fraction concentration provided by the vendor)

$C_{\text {PEG-4MAL }} 4.0 \%$ (wt/vol) (desired final PEG-4MAL density)

$C_{\mathrm{RGD}} 2.0 \mathrm{mM}$ (desired final adhesive ligand density)

$X_{\text {PEG-4MAL }} 0.4$ (volume fraction of PEG-4MAL in the hydrogel) 
$X_{\mathrm{RGD}} 0.2$ (volume fraction of RGD in the hydrogel)

$X_{\mathrm{GPQ}-\mathrm{W}} 0.2$ (volume fraction of GPQ-W in the hydrogel)

\section{Procedure}

1. Determine the volume needed to reconstitute $m_{\mathrm{PEG}-4 \mathrm{MAL}}\left(V_{\mathrm{PEG}-4 \mathrm{MAL}}\right)$ if the amount of PEG-4MAL is $100 \mathrm{mg}$ :

$$
V_{\mathrm{PEG}-4 \mathrm{MAL}}=m_{\mathrm{PEG}-4 \mathrm{MAL}} \times \frac{X_{\mathrm{PEG}-4 \mathrm{MAL}}}{C_{\mathrm{PEG}-4 \mathrm{MAL}}}=1.0 \mathrm{~mL}
$$

where $V=$ volume and $m=$ mass.

2. Determine the final molarity of the maleimide groups $\left(M_{\mathrm{Mal}}\right)$ on the basis of $m_{\mathrm{PEG}-4 \mathrm{MAL}}$ and $V_{\mathrm{PEG}-4 \mathrm{MAL}}$ :

$$
M_{\mathrm{MAL}}=m_{\mathrm{PEG}-4 \mathrm{MAL}} \times \frac{4 \times X_{\mathrm{PEG}-4 \mathrm{MAL}} \times P_{\mathrm{PEG}-4 \mathrm{MAL}}}{\mathrm{MW}_{\mathrm{PEG}-4 \mathrm{MAL}} \times V_{\mathrm{PEG}}-4 \mathrm{MAL}}=6.98 \mathrm{mM}
$$

3. Determine the volume needed to reconstitute the ( $\left.V_{\mathrm{RGD}}\right) \mathrm{RGD}$ and ( $V_{\mathrm{GPQ}}$-W $\mathrm{GPQ}-\mathrm{W}$ :

$$
V_{\mathrm{RGD}}=V_{\mathrm{PEG}-4 \mathrm{MAL}} \times \frac{\mathrm{XRGD}\left(\text { or } X_{\mathrm{GPQ}}-\mathrm{W}\right)}{X_{\mathrm{PEG}}-4 \mathrm{MAI}}=V_{\mathrm{GPQ}}-\mathrm{W}=0.5 \mathrm{~mL}
$$

4. Determine the mass of RGD ( $\left.m_{\mathrm{RGD}}\right)$ needed to obtain $C_{\mathrm{RGD}}$ :

$$
m_{\mathrm{RGD}}=C_{\mathrm{RGD}} \times \frac{\mathrm{MW}_{\mathrm{RGD}} \times V_{\mathrm{RGD}}}{P_{\mathrm{RGD}} \times X_{\mathrm{RGD}}}=4.06 \mathrm{mg}
$$

5. Determine the final molarity of free maleimide groups ( $\left.M_{\text {MAL-GPQ-W }}\right)$ after accounting for maleimide groups reacted with RGD:

$$
M_{\mathrm{MAL}-\mathrm{GPQ}}-\mathrm{W}=M_{\mathrm{MAL}}-C_{\mathrm{RGD}}=4.98 \mathrm{mM}
$$

6. Determine the mass of GPQ-W $\left(m_{\mathrm{GPQ}-\mathrm{W}}\right)$ needed to react with $M_{\mathrm{MAL}-\mathrm{GPQ}-\mathrm{W}}$ :

$$
m_{\mathrm{GPQ}}-\mathrm{W}=\mathrm{M}_{\mathrm{MAL}}-\mathrm{GPQ}-\mathrm{W} \times \frac{M_{\mathrm{WGPQ}} \times V_{\mathrm{GPQ}}-\mathrm{W}}{2 \times P_{\mathrm{GPQ}}-\mathrm{W} \times X_{\mathrm{GPQ}-\mathrm{W}}}=13.27 \mathrm{mg}
$$




\section{Related links}

Key reference using this protocol

Cruz-Acuña, R. et al. Nat. Cell Biol., 1326-1335 (2017) https://doi.org/10.1038/ncb3632. 


\section{Preparation of hydrogel precursor solutions}

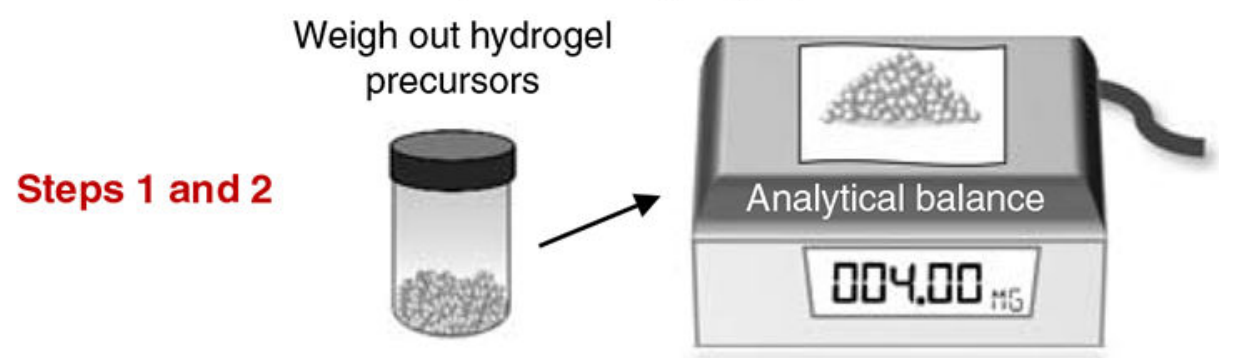

Reconstitute

hydrogel precursors
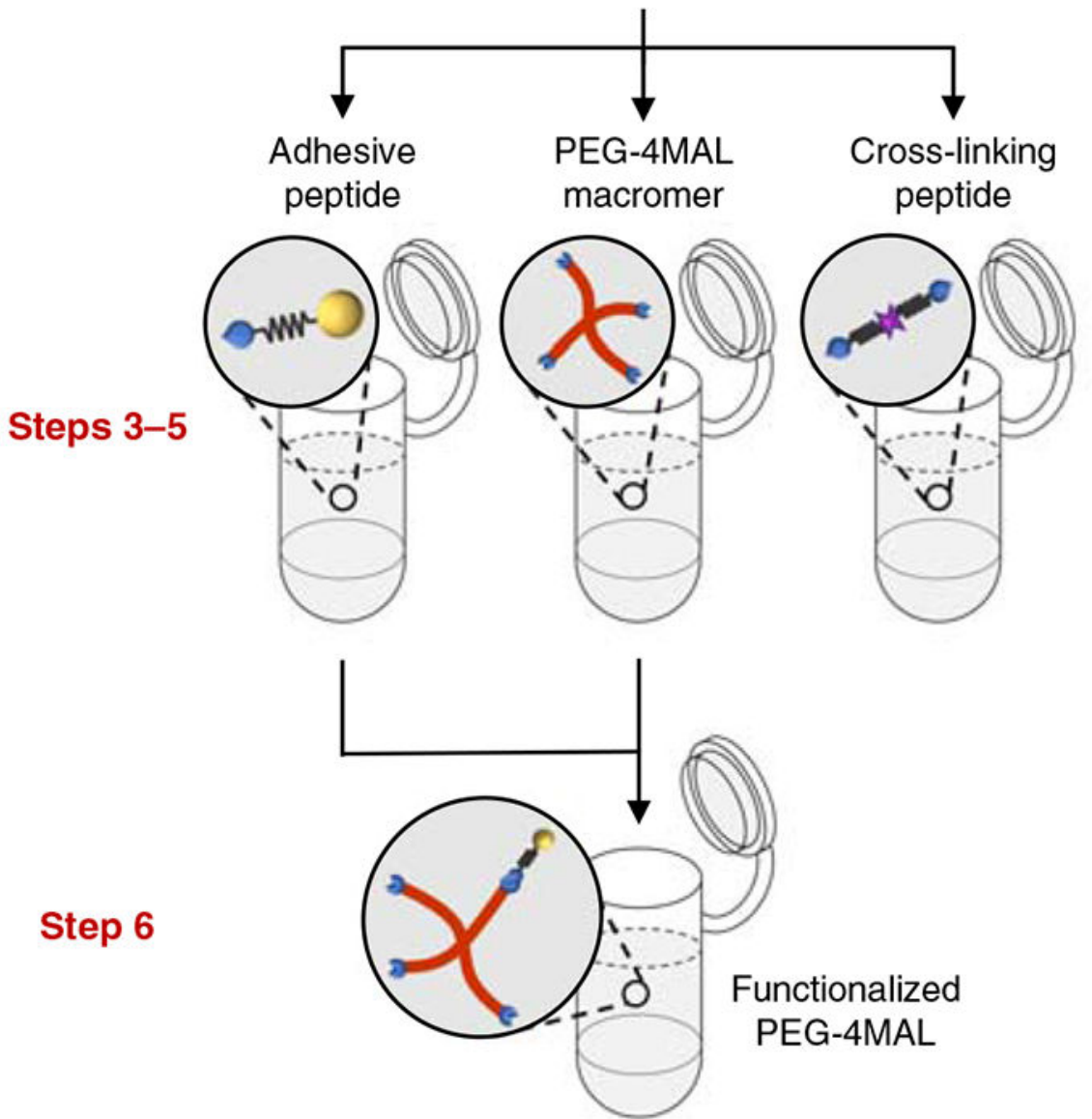

Fig. 1 |. Preparation of the solutions for the precursors of the PEG-4MAL hydrogel.

Hydrogel precursor solutions are reconstituted in separate tubes, and the functionalized PEG-4MAL macromer is produced by mixing the PEG-4MAL macromer solution with the adhesive ligand solution. The relevant steps of the protocol are highlighted in red. Adapted with permission from Cruz-Acuña et al. ${ }^{16}$, Springer Nature Limited. 
a

Human spheroid suspension in functionalized PEG-4MAL

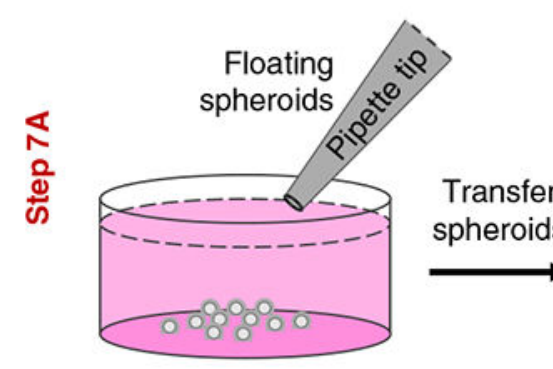

Functionalized PEG-4MAL

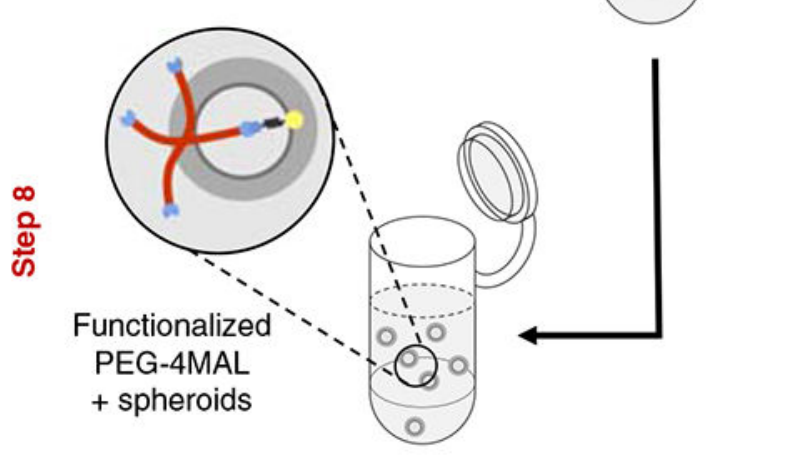

b

Synthetic hydrogel casting and organoid generation

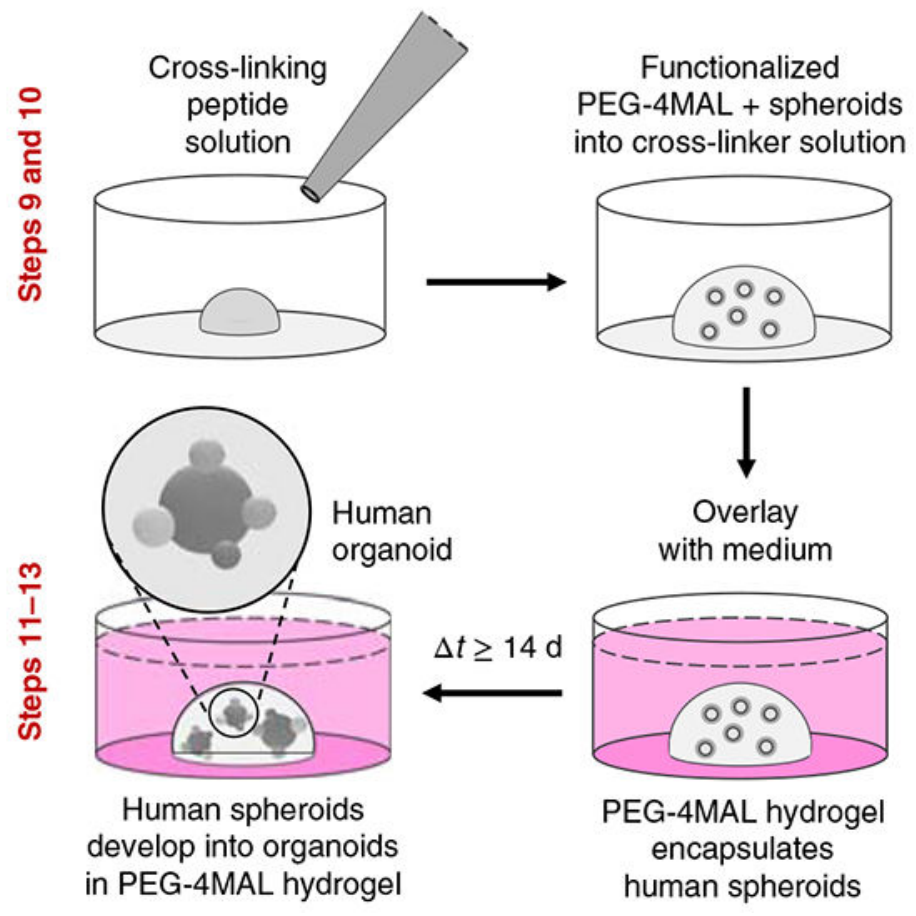

Fig. 2 |. Synthesis of the PEG-4MAL hydrogel and organoid generation.

a, Floating, hPSC-derived human spheroids (generated as previously described ${ }^{1,2}$ ) are collected and mixed with a solution of functionalized PEG-4MAL macromer. $\mathbf{b}$,

PEG-4MAL hydrogel is cast by pipetting the mixture of functionalized PEG-4MAL and spheroids into the cross-linker solution. Encapsulated spheroids expand and develop into human organoids. The relevant steps of the protocol are highlighted in red. Adapted with permission from Cruz-Acuña et al. ${ }^{16}$, Springer Nature Limited. 


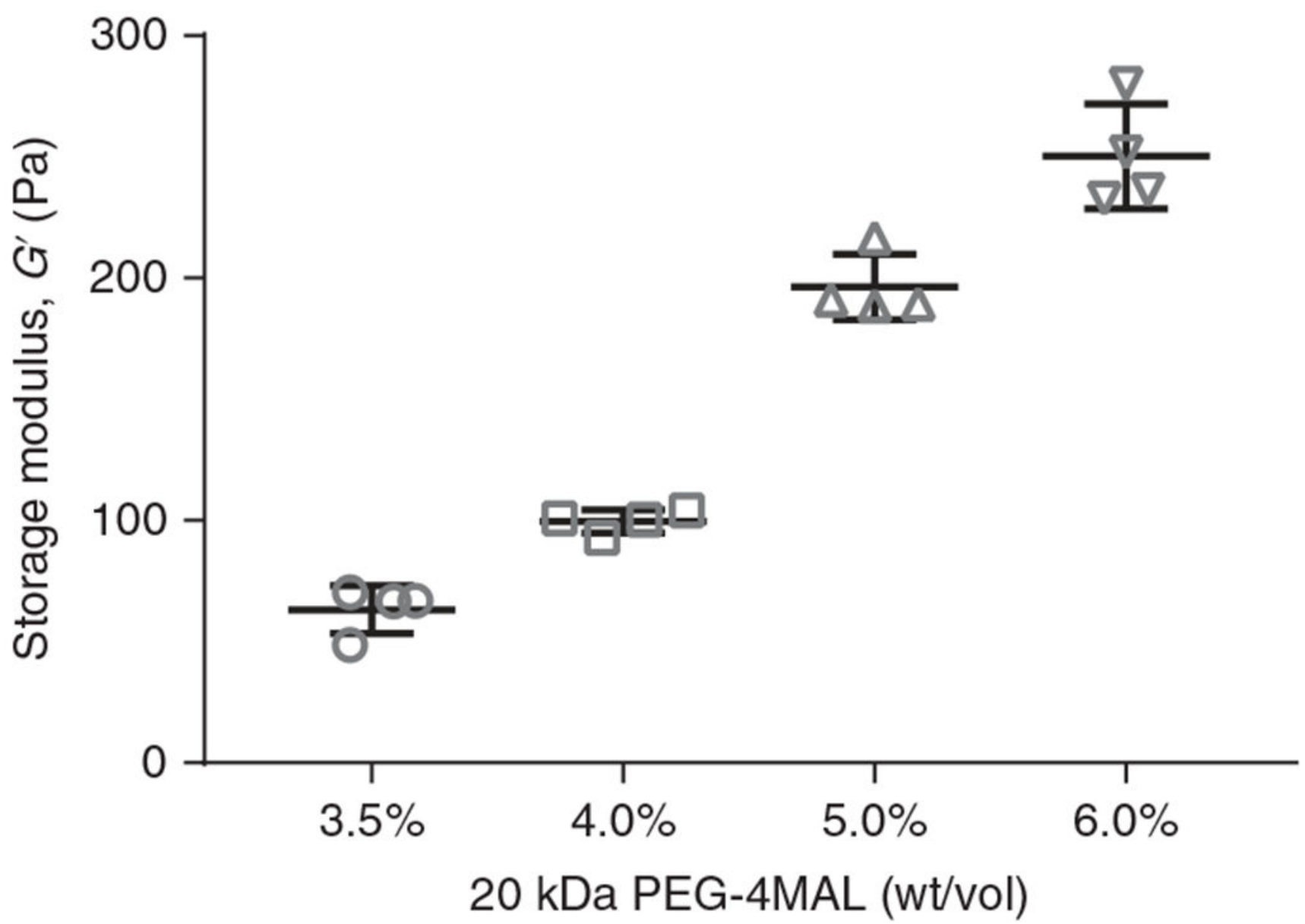

Fig. 3 |. Typical results of rheometric characterization of PEG-4MAL hydrogels. The relationship between polymer density (wt \%) and storage modulus of 20-kDa PEG-4MAL hydrogels with constant biochemical properties (2.0 mM RGD, using a GPQ-W cross-linker). Data are presented as mean \pm SEM; $n=4$ independently prepared hydrogels per condition. 


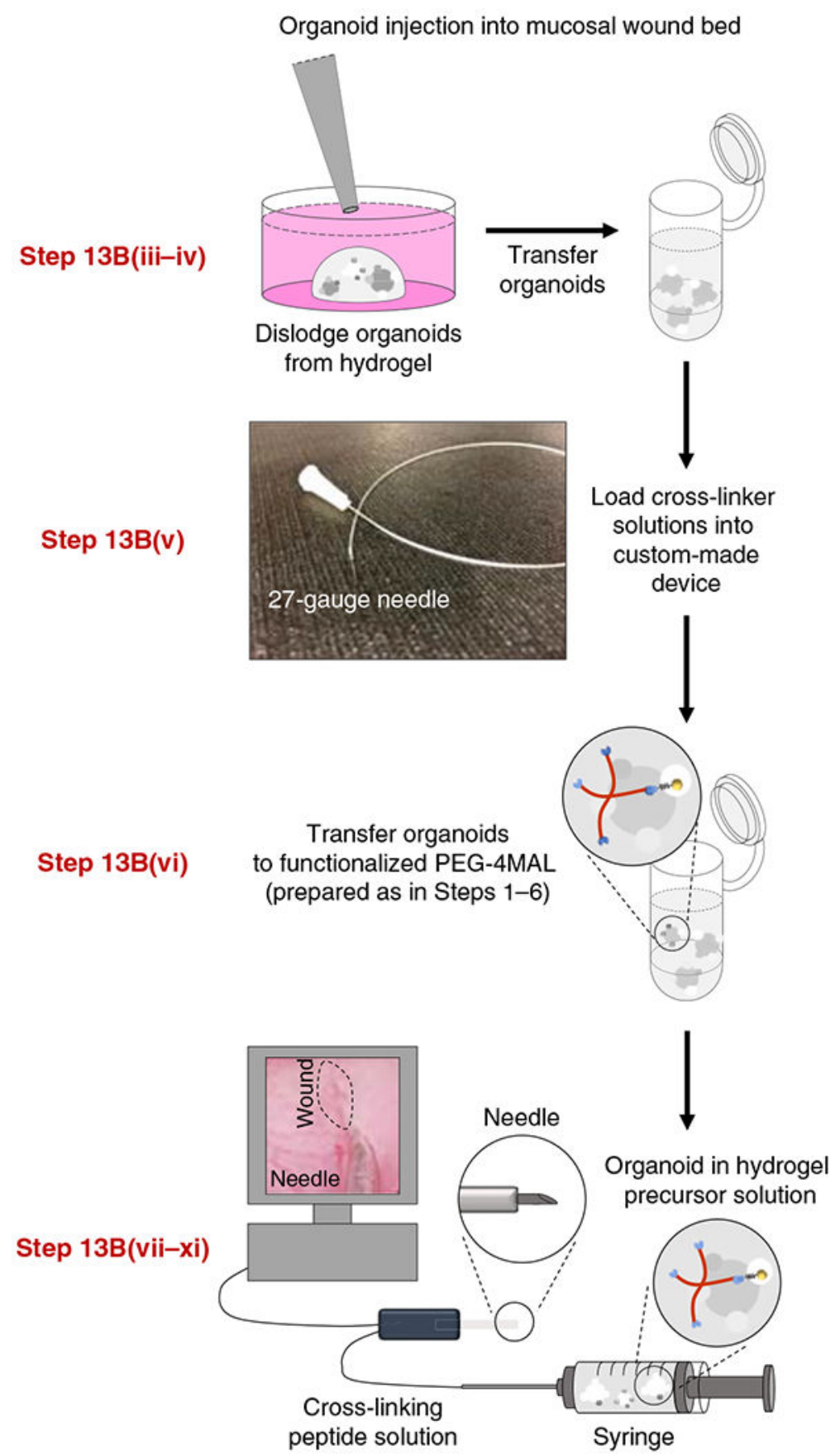

Fig. 4 |. Preparation of PEG-4MAL hydrogel-generated organoids and setup for mucosal injection.

HIOs generated in PEG-4MAL hydrogels are recovered from the matrix, mixed with hydrogel precursor solutions, and injected underneath the submucosal wounds using a custom-made device (comprising a $10-\mathrm{cm}$ piece of intramedic polyethylene tube with a $27-$ gauge needle at each end) via a colonoscope. The relevant steps of the protocol are highlighted in red. Adapted with permission from Cruz-Acuña et al. ${ }^{16}$, Springer Nature Limited. 
a

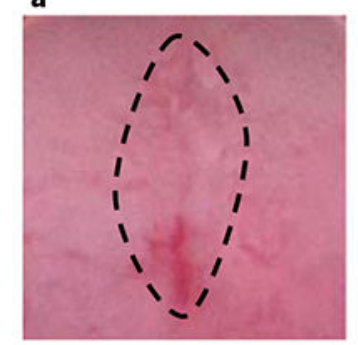

b

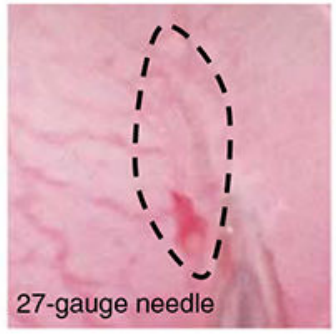

c

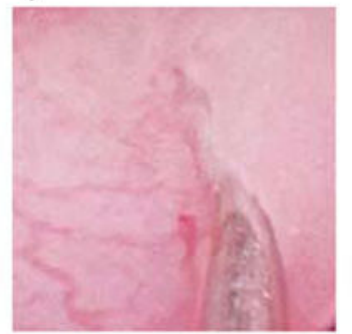

d

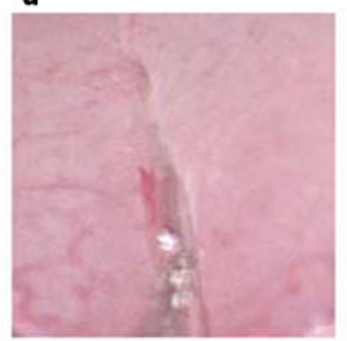

e

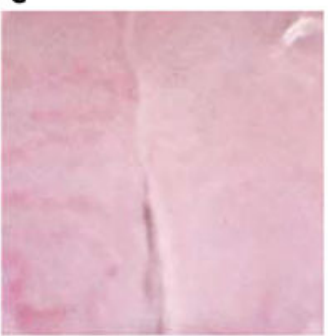

Fig. 5 |. PEG-4MAL hydrogel serves as an injectable delivery vehicle in a colonic mucosal wound model.

a, A wound is observed through the camera. b, The needle is inserted at the distal end of the wound by carefully penetrating the mucosa. $\mathbf{c}, \mathbf{d}$, The contents of the custom-made device are injected into the site. e, The needle is removed. The dotted line highlights the wound. The images show a 27 -gauge needle (o.d. $=0.41 \mathrm{~mm}$ ). All experiments with mice were performed while following national and institutional guidelines. 

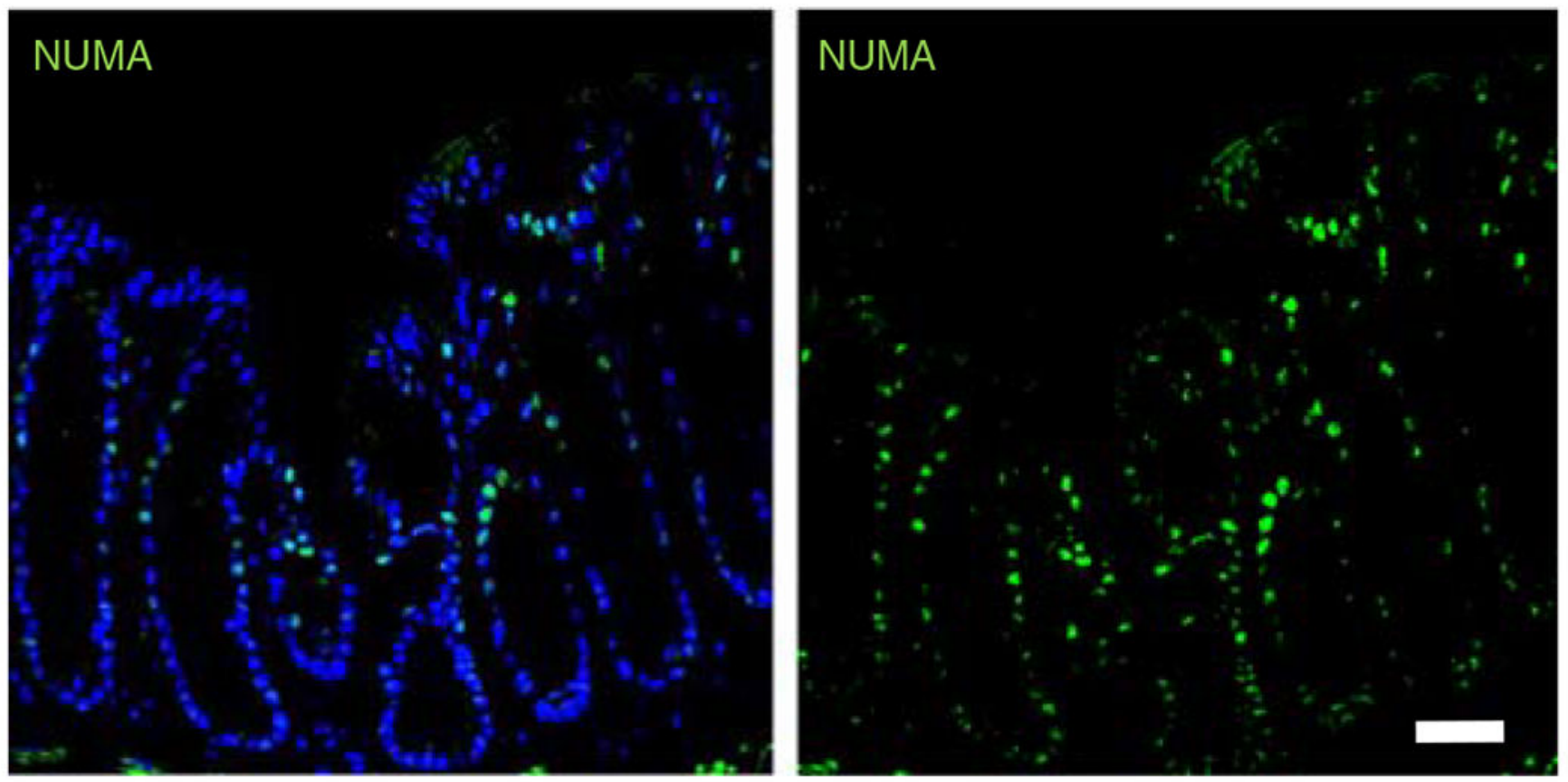

Fig. 6 |. PEG-4MAL hydrogel promotes HIO engraftment into mucosal wounds.

Fluorescence microscopy images of murine colonic tissue at the wound site labeled for human cell nuclei (NUMA, EMD Millipore, cat. no. MAB1281, overnight incubation at 1:100; followed by donkey anti-mouse Alexa Fluor 488, Jackson ImmunoResearch, cat. no. 715-545-150, 1-h incubation at 1:2,000; green) taken 4 weeks post delivery. DAPI (blue; 1:1,000), was used as counterstain. Scale bar, $100 \mu \mathrm{m}$. All experiments with mice were performed while following national and institutional guidelines. These data are representative of the results we saw after injection of hydrogels containing HIOs in two independent studies ( $n=4$ mice per condition, five injections per mouse). Further results from this study are published in ref. ${ }^{18}$ Samples were imaged using a Zeiss Axiovert 35 microscope and analyzed with LAS X software (Leica Microsystems). 
a

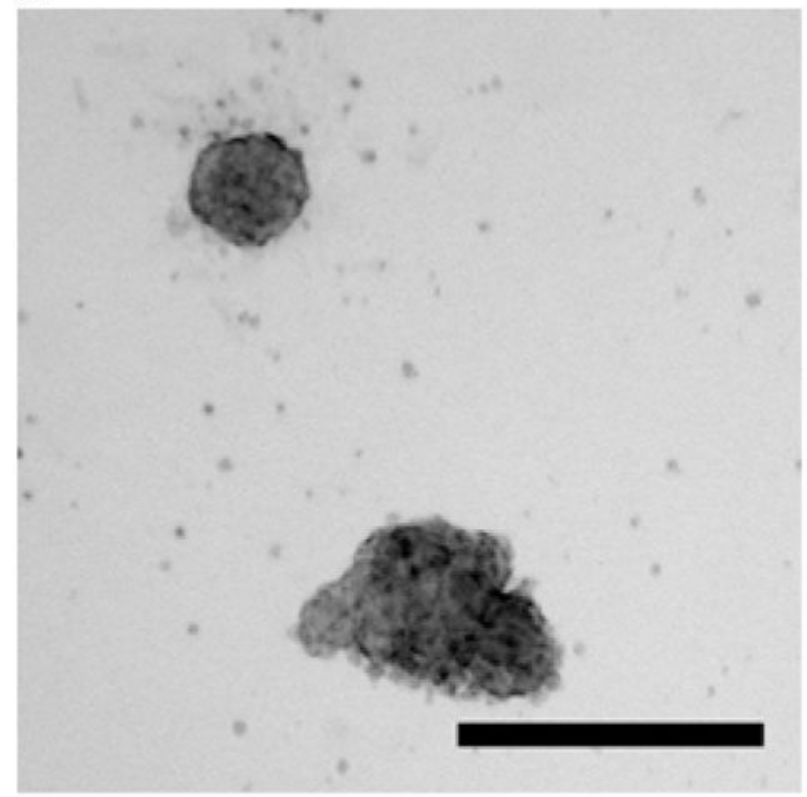

C

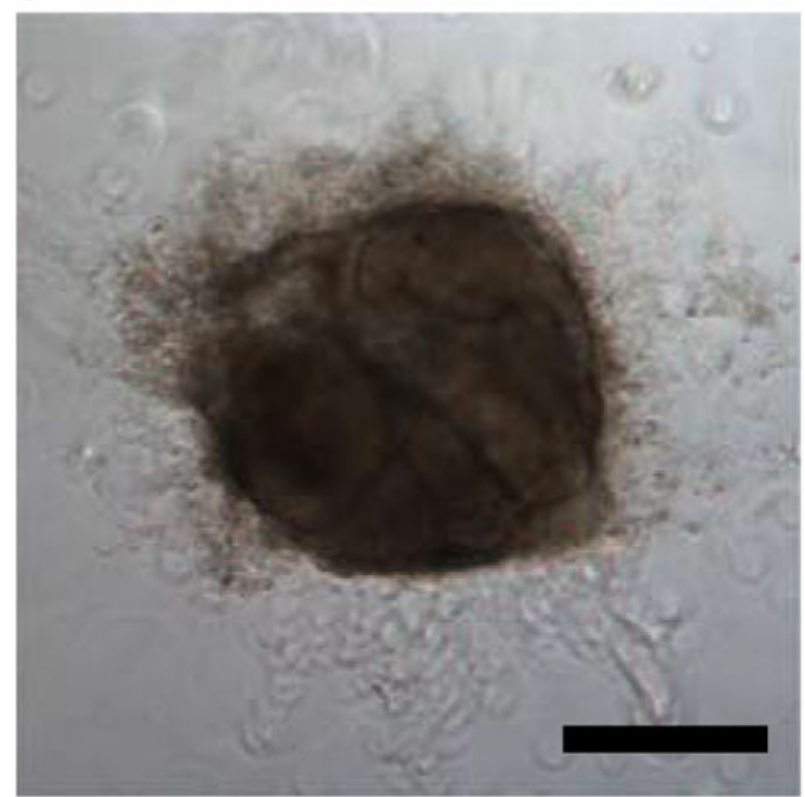

b

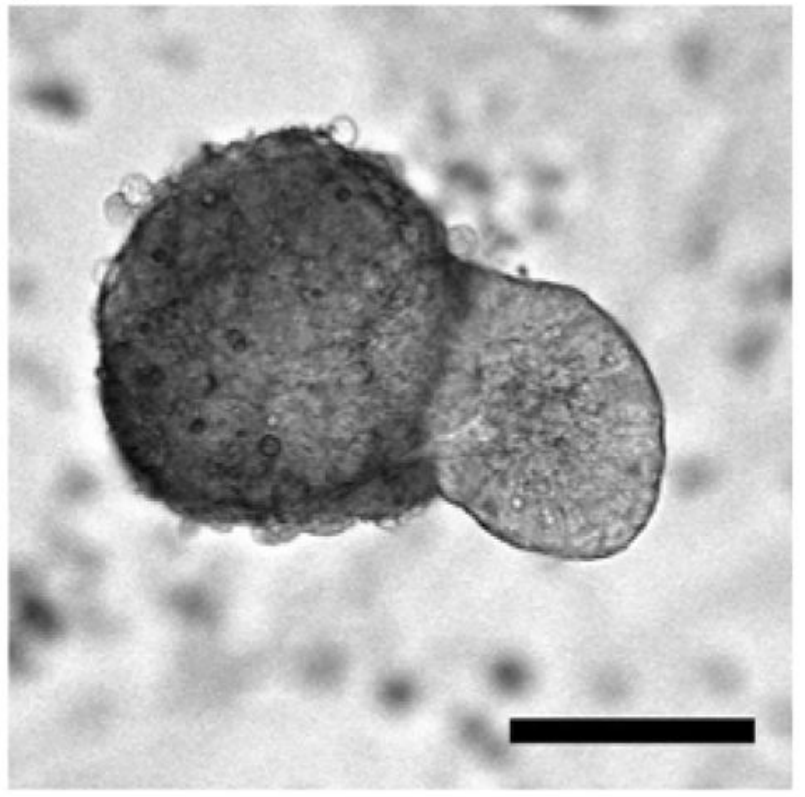

d

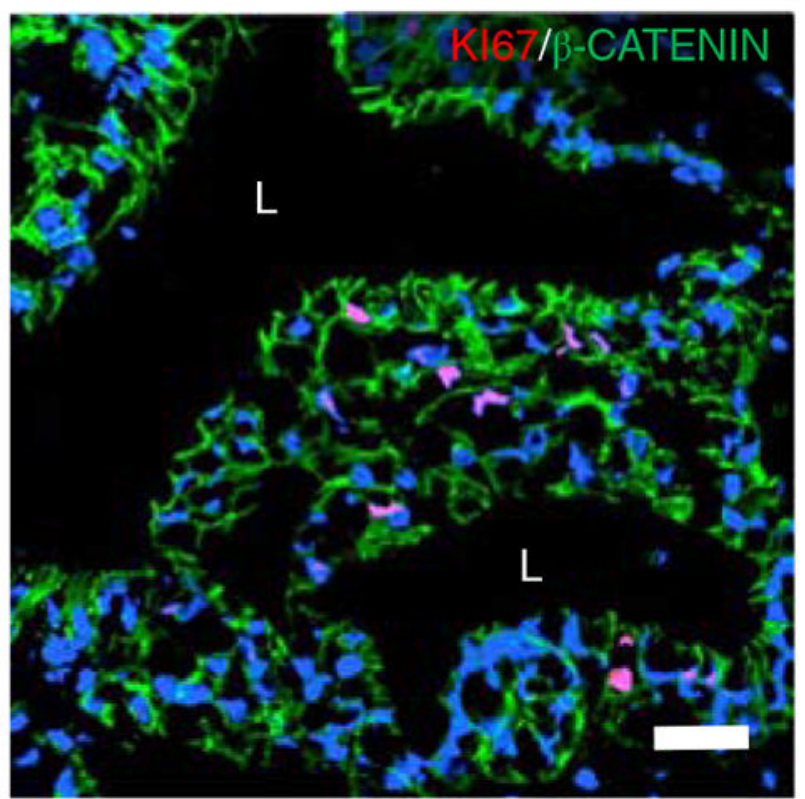

Fig. 7 |. PEG-4MAL hydrogel supports hPSC-derived intestinal spheroid development into HIOs.

a-c, Transmitted light microscopy images of hiPSC-derived HIOs generated within $4.0 \%$ (wt/vol) PEG-4MAL-RGD hydrogels $1 \mathrm{~d}(\mathbf{a}), 5 \mathrm{~d}$ (b), and $21 \mathrm{~d}$ (c) after encapsulation. c, $\mathrm{HIO}$ in has undergone two passages before this time point. Scale bars, $100 \mu \mathrm{m}(\mathbf{a}, \mathbf{b}) ; 500 \mu \mathrm{m}$ (c). d, Fluorescence microscopy image of a HIO taken $21 \mathrm{~d}$ after encapsulation in a $4.0 \%$ (wt/vol) PEG-4MAL-RGD hydrogel. HIO was labeled for cell-cell junctions ( $\beta$-catenin (green), BD Biosciences, cat. no. 610153, overnight incubation at 1:100, followed by goat 
anti-mouse Alexa Fluor 488, Thermo Fisher, cat. no. A-11017, 1-h incubation at 1:2,000, and proliferative cells (KI67 (red), Abcam, cat. no. ab15580), overnight incubation at 1:100, followed by donkey anti-rabbit Cy3, Jackson ImmunoResearch, cat. no. 711-165-152, 1-h incubation at 1:2,000). DAPI (blue; 1:1,000) was used as counterstain. ' $\mathrm{L}$ ' indicates HIO lumen. Scale bar, $100 \mu \mathrm{m}$. Samples were imaged using a Zeiss Axiovert 35 microscope and analyzed with LAS X software (Leica Microsystems). 
Table 1 |

Troubleshooting table

\begin{tabular}{|c|c|c|c|}
\hline Step & Problem & Possible reason & Solution \\
\hline \multirow[t]{4}{*}{$13 \mathrm{~A}(\mathrm{vii})$} & $\begin{array}{l}\text { Spheroids do not } \\
\text { develop into HOs }\end{array}$ & Insufficient number of medium changes & Change the medium every $3-4 \mathrm{~d}$ \\
\hline & & $\begin{array}{l}\text { Absence of growth factors/low growth } \\
\text { factor activity }\end{array}$ & Ensure that the growth factors are freshly added \\
\hline & $\begin{array}{l}\text { Spheroids sink to the } \\
\text { bottom of the dish and } \\
\text { fail to develop into HOs }\end{array}$ & $\begin{array}{l}\text { The hydrogel is degrading too quickly or is } \\
\text { not properly polymerized }\end{array}$ & $\begin{array}{l}\text { Reduce the spheroid density per hydrogel Increase the } \\
\text { frequency of passages to every } 5-7 \mathrm{~d}\end{array}$ \\
\hline & & $\begin{array}{l}\text { Spheroids were too close to the bottom of } \\
\text { the dish during hydrogel cross-linking }\end{array}$ & $\begin{array}{l}\text { During hydrogel cross-linking, flip the plate upside- } \\
\text { down to ensure that spheroids are not too close to the } \\
\text { bottom of the dish }\end{array}$ \\
\hline \multirow[t]{4}{*}{$\begin{array}{l}11 \\
13 \mathrm{~B}(\mathrm{xi})\end{array}$} & Hydrogels do not form & $\begin{array}{l}\text { Inadequate } \mathrm{pH} \text { adjustment of the peptide } \\
\text { solutions }\end{array}$ & Adjust the $\mathrm{pH}$ to 7.4 \\
\hline & & $\begin{array}{l}\text { The purity level of the peptides was not } \\
\text { considered when calculating mass }\end{array}$ & $\begin{array}{l}\text { Consider the peptide purity provided by the vendor as } \\
\text { shown in Box } 1\end{array}$ \\
\hline & & & $\begin{array}{l}\text { Determine the peptide purity by UV absorption } \\
\text { spectroscopy, as previously described }{ }^{29}\end{array}$ \\
\hline & & $\begin{array}{l}\text { Incomplete tissue penetration of the needle } \\
\text { bevel at the wound site (only for Step } \\
13 \mathrm{~B}(\mathrm{xi}) \text { ) }\end{array}$ & $\begin{array}{l}\text { Penetrate the tissue slightly past the needle bevel to } \\
\text { ensure full delivery of hydrogel solution }\end{array}$ \\
\hline \multirow[t]{3}{*}{ 13B(xii) } & $\begin{array}{l}\text { Low or no HO } \\
\text { engraftment into host } \\
\text { tissue }\end{array}$ & $\begin{array}{l}\text { Settling of HOs to one end of the syringe } \\
\text { due to gravity }\end{array}$ & Shake the syringe gently before each injection \\
\hline & $\begin{array}{l}\text { Clogging in the tubing } \\
\text { caused by hydrogel } \\
\text { cross-linking }\end{array}$ & $\begin{array}{l}\text { Untimely mixing of the hydrogel precursor } \\
\text { solutions in the tube before injection }\end{array}$ & $\begin{array}{l}\text { Verify that there is no leakage from the syringe into } \\
\text { the tube before injections }\end{array}$ \\
\hline & & The $\mathrm{pH}$ is too high & $\begin{array}{l}\text { Reduce the } \mathrm{pH} \text { of the cross-linker solution to delay the } \\
\text { reaction during injection }\end{array}$ \\
\hline
\end{tabular}

